\title{
FREQUENCY RESPONSE OF A SUPPORTED THERMOCOUPLE WIRE: EFFECTS OF AXIAL CONDUCTION
}

Progress Report October 1990 - March 1991

\section{J. Forney}

School of Chemical Engineering Georgia Institute of Technology Atlanta, Georgia 30332

\section{E. L. Meeks}

Microelectronics Research Center Georgia Institute of Technology Atlanta, Georgia 30332

\section{G. C. Fralick}

Research Sensor Technology Branch NASA - Lewis Research Center Cleveland, Ohio 44135
Lewis

SPAN?

$1 N-35-C K$ 5409

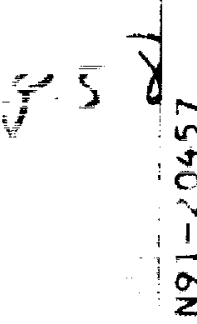

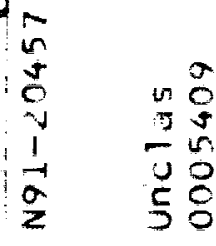

Sponsored by

ARNOLD ENGINEERING DEVELOPMENT CENTER Arnold Air Force Base, Tennessee 37389 under

NASA Cooperative Agreement NCC 3-135

Georgia Institute of Technology

$$
\text { April, } 1991
$$



$\bar{C}$ -

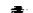

$=$

-

$=$ $-$ 
Frequency Response of a Supported Thermocouple Wire :

Effects of Axial Conduction

\author{
L. J. Forney \\ School of Chemical Engineering \\ Georgia Institute of Technology \\ Atlanta, Georgia 30332 \\ E. L. Meeks \\ Microelectronics Research Center \\ Georgia Institute of Technology \\ Atlanta, Georgia 30332 \\ G. C. Fralick \\ Research Sensor Technology Branch \\ NASA - Lewis Research Center \\ Cleveland, Ohio 44135
}

Georgia Institute of Technology

April, 1991 


\section{ABSTRACT}

Theoretical expressions are derived for the steady-state frequency response of a supported thermocouple wire. In particular, the effects of axial heat conduction are demonstrated for both a supported one material wire and a two material wire with unequal material properties across the junction. For the case of a one material supported wire, an exact solution is derived which compares favorably with an approximate expression that only matches temperatures at the support junction. Moreover, for the case of a two material supported wire, an analytical expression is derived that closely correlates numerical results.

Experimental data were taken with a type $\mathrm{K}$ supported thermocouple. The test thermocouple was constructed with dimensions to demonstrate the effects of axial heat conduction assuming constant physical properties across the junction. 



\section{INTRODUCTION}

The evaluation of jet engine performance and fundamental studies of combustion phenomena depend on the measurement of turbulent fluctuating temperatures of the gas within the engine (Dils and Follansbee, 1976). Historically, these temperatures have been measured with thermocouples. The design of a thermocouple represents a compromise between accuracy, ruggedness and rapidity of response.

For example, the measurement of fluctuating temperatures in the high speed exhaust of a gas turbine engine combustor is required to characterize the local gas density gradients or convective heat transfer (Fralick, 1985). Although thermocouples are suitable for the measurement of high frequency temperature fluctuations $(<1 \mathrm{KHz})$ in a flowing gas or liquid, the measured signal must be compensated since the frequency of the time dependent fluid temperature is normally much higher than the corner frequency of the thermocouple probe (Scadron and Warshawsky, 1952). Moreover, the amplitude and phase angle of the thermocouple response may be attenuated by axial heat conduction for rugged thermocouples of finite length (Elmore, et al.; 1983, 1986).

In the present study, the theoretical steady-state frequency response of a supported thermocouple wire has been calculated to include the effects of axial heat conduction. These solutions, which represent an extension of earlier work (Forney and Fralick, 1991) are derived for both a supported thermocouple wire 
with equal physical properties across the junction (e.g., roughly the same thermoconductivity, etc.) and a supported wire with unequal properties across the junction. Solutions are presented in the form of the amplitude ratio and phase angle for both cases.

\section{THEORY FOR ONE MATERIAL THERMOCOUPLE}

The steady-state frequency response of a thermocouple wire will be developed with the following assumptions: (a) the amplitude of the fluctuating fluid temperature is small relative to the mean absolute temperature (b) the thermocouple dimensions are small relative to the size of the turbulent eddies or

enclosure dimensions (c) radial temperature gradients in a wire cross section can be neglected and (d) radiative heat transfer can be neglected relative to conduction and convection.

In this section the geometry of Fig. 1 is considered where the material properties of thermal conductivity $k$, specific heat $c$ and wire density $\rho$ are assumed to be equal on both sides of the thermocouple junction. If the probe is immersed in a flowing fluid, the expression for the local conservation of energy in the thermocouple wire becomes (Scadron and Warshawsky, 1952)

$$
\frac{\partial T_{W}}{\partial t}=\alpha \frac{\partial^{2} T_{w}}{\partial x^{2}}+\frac{4 h}{\rho c D}\left(T_{z}-T_{W}\right)
$$


where $\alpha=k / \rho c$ is the thermal diffusivity of the wire, $\mathrm{T}_{\mathrm{B}}$ is the ambient fluid temperature, $h$ is the convective heat transfer coefficient, $D$ is the wire diameter and $T_{w}$ is the local wire temperature measured along the axis at a distance $x$ from the centerline (Fig. 1.)

The wire and fluid temperatures are measured relative to the mean fluid temperature $T_{0}$. The ambient fluid temperature is taken to be a mean temperature together with a sinusoidal varying deviation from the mean,

$$
T_{g}(t)=T_{0}+T_{f} e^{i \omega t}
$$

where $\omega$ is the angular frequency of the ambient temperature. Since Eq. (1) is linear, we now seek a solution for the local wire temperature of the form (Hildebrand, 1976)

$$
T_{w}=T_{0}+\vec{T}_{\omega}(x) e^{i \omega t}
$$

Referencing all temperatures with respect to the mean gas temperature $T_{0}$ and normalizing with respect to the amplitude of the fluctuating ambient fluid temperature $T_{f}$, one defines a local normalized steady-state frequency response $\vec{T}(x)$ for the thermocouple wire of the form

$$
\frac{T_{w}-T_{0}}{T_{f}}=\frac{\vec{T}_{\omega}(x) e^{i \omega t}}{T_{f}}=\vec{T}(x) e^{i \omega !}
$$


Substituting Eqs. (2) and (3) into Eq. (1), one obtains an ordinary differential equation of the form

$$
i \omega T=\alpha \frac{d^{2} T}{d x^{2}}+\omega_{n}(1-T)
$$

where $T=\vec{T}(x)$ is the frequency response and the vector notation will be dropped for simplicity. Thus, for the geometry of fig. 1, one seeks a solution to the nonhomogeneous linear second order differential equation for the dependent variable T of the form (Forney and Fralick, 1990)

$$
\gamma T^{\prime \prime}-G(\omega) T=-1
$$

The general solution to Eq.(6) can be written in the form (Hildebrand, 1976)

$$
T(x)=A \sinh q \mathrm{x}+\mathrm{B} \cosh q \mathrm{x}+\frac{1}{G(\omega)}
$$

where the parameters in Eqs. (5) and (6) are defined as

$$
\omega_{n}=\frac{4 h}{\rho c D}, \quad \gamma=\frac{\alpha}{\omega_{n}}, \quad G(\omega)=1+i\left(\frac{\omega}{\omega_{n}}\right)
$$

while in Eq. (7) the constants $A$ and $B$ are complex, $1 / G(\omega)$ represents the particular solution and the parameter 


$$
q=\sqrt{\frac{G(\omega)}{\gamma}}
$$

\section{Approximate Solution}

Assuming that the material properties are constant across the junction and that the wire diameters are $D_{1}$ and $D_{2}$ in regions 1 and 2, respectively, Eq. (6) is subject to the boundary conditions

$$
\begin{aligned}
& T_{1}(l)=T_{2}(l)=T_{a} \\
& T_{2}(l+L)=0 .
\end{aligned}
$$

In this case, we seek a simple approximate solution that neglects the heat transfer at the interface between regions 1 and 2 at $x= \pm l$ where the parameters in Eqs. (5) and (6) are defined in terms of the wire diameters in each region. A similar approach will be used in a later section to obtain an approximate solution for the case in which the material properties of the two elements of the thermocouple are distinctly different. Hence, in region 1

$$
\omega_{1}=\frac{4 h_{1}}{\rho c D_{1}}, \quad \gamma=\frac{\alpha}{\omega_{1}}, \quad G_{1}(\omega)=1+i\left(\frac{\omega}{\omega_{1}}\right)
$$

where $\omega_{1}$ is the natural frequency of the wire in region 1 of fig. 1. 
The solution to Eq. (6) for the one material wire on both sides of the junction in region 1 can be written in the form

$$
T_{1}(x)=A_{1} \sinh q_{1} x+B_{1} \cosh q_{1} x+\frac{1}{G_{1}(\omega)}
$$

Substituting $x= \pm l$ in Eq. (11), the boundary conditions in Eq. (9) yield values for the constants

$$
A_{1}=0, \quad B_{1}=\frac{1}{\cosh q_{1} l}\left(T_{a}-\frac{1}{G_{1}}\right) .
$$

Thus, one obtains a steady-state temperature distribution for the wire in region 1 of Fig. 1 in the form

$$
T_{1}(x)=\frac{1}{G_{1}}\left(1-\frac{\cosh q_{1} x}{\cosh q_{1} l}\right)+\mathrm{T}_{a}\left(\frac{\cosh q_{1} x}{\cosh q_{1} l}\right)
$$

We now seek a solution in region 2 that satisfies the boundary conditions of Eq. (9). Since the temperature is symmetric about $x=0$, it is convenient to define a continuous steady-state temperature distribution for the large wire of diameter $D_{2}$ over the entire region $-(l+L) \leq x \leq(l+L)$ or

$$
T_{2}(x)=\frac{1}{G_{2}}\left(1-\frac{\cosh q_{2} x}{\cosh q_{2}(l+L)}\right) .
$$


Since $T_{a}=T_{2}(l)$, one obtains the boundary value from Eq. (14)

$$
T_{a}=\frac{1}{G_{2}}\left(1-\frac{\cosh q_{2} l}{\cosh q_{2}(l+L)}\right) .
$$

Substituting the value for $T_{a}$ at $x=l$ of Eq. (15) into Eq. (13), the approximate temperature distribution in region 1 becomes

$$
T_{1}(x)=\frac{1}{G_{1}}\left(1-\frac{\cosh q_{1} x}{\cosh q_{1} l}\right)+\frac{1}{G_{2}}\left(1-\frac{\cosh q_{2} l}{\cosh q_{2}(l+L)}\right) \frac{\cosh q_{1} x}{\cosh q_{1} l}
$$

Thus, the approximate frequency response at the thermocouple junction $(x=0)$ for the one material wire becomes

$$
T_{1}(0)=\frac{1}{G_{1}}\left(1-\frac{1}{\cosh q_{1} l}\right)+\frac{1}{G_{2}}\left(1-\frac{\cosh q_{2} l}{\cosh q_{2}(l+L)}\right) \frac{1}{\cosh q_{1} l}
$$

The steady-state frequency at the thermocouple junction $x=0$ is normally characterized graphically in the form

$$
T(0)=|T(0)| e^{i \oplus}
$$


where $|T(0)|$ is the amplitude ratio and $\Phi$ is the phase angle. In the latter case, the phase angle in degrees is

$$
\Phi=57.3 \tan ^{-1}\left[\frac{\operatorname{Im} T(0)}{\operatorname{Re} T(0)}\right]
$$

where $\operatorname{Im}[T(0)]$ and $\operatorname{Re}[T(0)]$ are the imaginary and real parts of $T(0)$, respectively.

\section{Exact Solution}

If the boundary conditions listed in Eq.(9) include equal rates of conductive heat transfer at the interface between the thermocouple and support wires at $x= \pm l$, the exact solution is subject to

$$
\begin{gathered}
T_{1}(l)=T_{2}(l) \\
k D_{1}^{2} \frac{d T_{1}(l)}{d x}=k D_{2}^{2} \frac{d T_{2}(l)}{d x} \\
T_{2}(l+L)=0 .
\end{gathered}
$$

Since the solution to Eq. (6) in region 1 is of the form

$$
T_{1}(x)=A_{1} \sinh q_{1} x+B_{1} \cosh q_{1} x+\frac{1}{G_{1}}
$$


where by symmetry $T_{1}(l)=T_{1}(-l)$, one obtains $A_{1}=0$. Thus, the form of the solution in region 1 becomes

$$
T_{1}(\mathrm{x})=B_{1} \cosh q_{1} \mathrm{x}+\frac{1}{G_{1}}
$$

For region 2, where the spatial coordinate is in the range $l \leq x \leq(l+L)$, it is convenient to write

$$
T_{2}(x)=A_{2} \sinh q_{2}(l+L-x)+B_{2} \cosh q_{2}(l+L-x)+\frac{1}{G_{2}}
$$

From the boundary condition $T_{2}(l+L)=0$, one obtains $B_{2}=-1 / G_{2}$ or the form of the solution in region 2 becomes

$$
T_{2}(x)=A_{2} \sinh q_{2}(l+L-x)+\frac{1}{G_{2}}\left[1-\cosh q_{2}(l+L-x)\right] .
$$

Substituting Eqs. (22) and (23) into boundary condition (20a), one obtains a linear equation for the constants $B_{1}, A_{2}$ or

$$
B_{1} \cosh q_{1} l-A_{2} \sinh q_{2} L=\frac{1}{G_{2}}\left[1-\cosh q_{2} L\right]-\frac{1}{G_{1}}
$$

Similarly, substituting Eqs. (22) and (23) into boundary condition (20b), one obtains a second linear equation for $B_{1}, A_{2}$ or 


$$
B_{1} Q \sinh q_{1} l+A_{2} \cosh q_{2} L=\frac{1}{G_{2}} \sinh q_{2} L
$$

where the complex constant $Q$ is defined as

$$
Q=\frac{D_{1}^{2} q_{1}}{D_{2}^{2} q_{2}}
$$

Solving Eqs. (25) and (26) for $B_{1}$ and $A_{2}$ one obtains the determinate system

$$
B_{1}=\frac{\left|\begin{array}{cc}
\frac{1}{G_{2}}\left[1-\cosh q_{2} L\right]-\frac{1}{G_{1}} & -\sinh q_{2} L \\
\frac{1}{G_{2}} \sinh q_{2} L & \cosh q_{2} L
\end{array}\right|}{D E T}
$$

and

$$
A_{2}=\frac{\left|\begin{array}{cc}
\cosh q_{1} l & \frac{1}{G_{2}}\left[1-\cosh q_{2} L\right]-\frac{1}{G_{1}} \\
2 \sinh q_{1} l & \frac{1}{G_{2}} \sinh q_{2} L
\end{array}\right|}{\mathrm{DET}}
$$

where the determinate in the denominator is equal to

$$
D E T=\cosh q_{1} l \cosh q_{2} L+Q \sinh q_{1} l \sinh q_{2} L
$$

Thus, solving for the constants $B_{1}$ and $A_{2}$ from Eqs. (28) and (29) and substituting 
$B_{1}$ into Eq. (22), one obtains an exact expression for the steady-state temperature distribution in the form

$$
T_{1}(x)=\frac{\left[\frac{1}{G_{2}}\left(\cosh q_{2} L-1\right)-\frac{1}{G_{1}} \cosh q_{2} L\right] \cosh q_{1} x}{\cosh q_{1} l \cosh q_{2} L+Q \sinh q_{1} l \sinh q_{2} L}+\frac{1}{G_{1}} .
$$

Thus, the steady-state frequency response at the thermocouple junction $x=0$ becomes

$$
T(0)=\frac{\frac{1}{G_{2}}\left(\cosh q_{2} L-1\right)-\frac{1}{G_{1}} \cosh q_{2} L}{\cosh q_{1} l \cosh q_{2} L+Q \sinh q_{1} l \sinh q_{2} L}+\frac{1}{G_{1}} .
$$

\section{THEORY FOR TWO MATERIAL THERMOCOUPLE}

Certain types of thermocouples have distinctly different material properties across the junction. For example, a copper-constantan thermocouple has a thermal conductivity on the copper side that is more than an order of magnitude larger than constantan. In this case, the expressions developed in the previous section for the frequency response are in error since unequal material properties would provide an asymmetric temperature profile.

Referring to fig. 2, the thermocouple schematic now has four distinct regions that are distinguished by either different wire diameters or physical properties. For example, on the left side of the schematic of fig. 1 the thermal conductivity, density 
and specific heat have the values $k_{1}, \rho_{1}$ and $c_{1}$, respectively, while on the right side of the schematic the material properties are $k_{2}, \rho_{2}$ and $c_{2}$.

\section{Temperature Distribution for Small Wire}

Since the differential equation describing the steady-state frequency response Eq. (6) applies to all regions of the schematic of fig. 1, the steady-state frequency response in regions 1 and 2 are given, respectively, by the expressions

$$
\begin{aligned}
& T_{1}(x)=A_{1} \sinh q_{1} x+B_{1} \cosh q_{1} x+\frac{1}{G_{1}} \\
& T_{2}(x)=A_{2} \sinh q_{2} x+B_{2} \cosh q_{2} x+\frac{1}{G_{2}} .
\end{aligned}
$$

Here, the four constants designated by A and B in Eqs. (33) and (34) are determined by the four boundary conditions

$$
\begin{gathered}
T_{1}(0)=T_{2}(0) \\
k_{1} D_{1}^{2} \frac{d T_{1}(0)}{d x}=k_{2} D_{1}^{2} \frac{d T_{2}(0)}{d x} \\
T_{1}(-l)=T_{\mathrm{a}} \\
T_{2}(l)=T_{\mathrm{b}} .
\end{gathered}
$$


Solving for the four values of the constants designated by A and B in Eqs. (33) and (34), one obtains an expression for the steady-state temperature distribution in region 1 of the form

$$
\begin{aligned}
& T_{1}(x)=\frac{1}{G_{1}}+\left\{\sinh q_{1}(x+l)\left[\left(\frac{1}{G_{2}}-\frac{1}{G_{1}}\right) \cosh q_{2} l+\left(T_{b}-\frac{1}{G_{2}}\right)\right]\right. \\
& \left.+\left(T_{a}-\frac{1}{G_{1}}\right)\left[Q_{t} \cosh q_{1} x \sinh q_{2} l-\sinh q_{1} x \cosh q_{2} l\right]\right\}\left(\frac{1}{\Delta_{1}}\right)
\end{aligned}
$$

and in region 2

$$
\begin{gathered}
T_{2}(x)=\frac{1}{G_{2}}+\left\{-Q_{t} \sinh q_{2}(l-x)\left[\left(\frac{1}{G_{2}}-\frac{1}{G_{1}}\right) \cosh q_{1} l-\left(T_{a}-\frac{1}{G_{1}}\right)\right]\right. \\
\left.+\left(T_{b}-\frac{1}{G_{2}}\right)\left[\cosh q_{2} x \sinh q_{1} l+Q_{t} \cosh q_{1} l \sinh q_{2} x\right]\right\}\left(\frac{1}{\Delta_{1}}\right) .
\end{gathered}
$$

Here, it should be noted that the two functions above can be obtained from each other by interchanging the subscripts 1 and 2, the values $a$ and $b$ and by changing the sign of $x$. Also, in Eqs. (36a) and (36b) the parameters

$$
\Delta_{1}=Q_{t} \cosh q_{1} l \sinh q_{2} l+\cosh q_{2} l \sinh q_{1} l
$$

and

$$
Q_{t}=\frac{k_{1} q_{1}}{k_{2} q_{2}}
$$




\section{Temperature Distribution for Large Wire}

In this section a solution is sought for the large wire in regions 3 and 4 of the schematic of fig. 2. To simplify the analysis, a continuous solution is derived for the steady-state frequency response over the entire region $-(l+L) \leq x \leq(l+L)$. This solution must satisfy the boundary conditions

$$
\begin{gathered}
T_{3}(0)=T_{4}(0) \\
k_{1} D_{3}^{2} \frac{d T_{3}(0)}{d x}=k_{2} D_{3}^{3} \frac{d T_{4}(0)}{d x} \\
T_{3}(-l-L)=0 \\
T_{4}(l+L)=0 .
\end{gathered}
$$

In principle, it is now possible to derive a continuous solution for $T_{3}(x)$ over the range $-(l+L) \leq x \leq 0$ and for $\mathrm{T}_{4}(\mathrm{x})$ over the range $0 \leq x \leq(l+L)$. These solutions are obtained from Eqs. (36a) and (36b) by replacing the subscripts $1 \rightarrow 3$ and $2 \rightarrow 4$ and by redefining the parameters $l \rightarrow l+L$ and setting the boundary conditions $T_{\mathrm{a}}=T_{\mathrm{b}}=0$. Moreover, the boundary condition $T_{\mathrm{a}}=T_{3}(-l)$ is obtained from the resulting expression for $T_{3}(x)$ by substituting $x=-l$ while the boundary condition $T_{b}=T_{4}(l)$. Thus, one obtains values for $T_{a}, T_{b}$ that appear in Eqs. (36a) and (36b) in the form 


$$
\begin{aligned}
& T_{a}=\frac{1}{G_{3}}+\left\{\sinh q_{3} L\left[\left(\frac{1}{G_{4}}-\frac{1}{G_{3}}\right) \cosh q_{4}(l+L)-\frac{1}{G_{4}}\right]\right. \\
& \left.-\frac{1}{G_{3}}\left[Q_{s} \cosh q_{3} I \sinh q_{4}(l+L)+\cosh q_{4}(l+L) \sinh q_{3} l\right]\right\}\left(\frac{1}{\Delta_{2}}\right)
\end{aligned}
$$

and

$$
\begin{aligned}
& T_{b}=\frac{1}{G_{4}}+\left\{Q_{s} \sinh q_{4} L\left[\left(\frac{1}{G_{3}}-\frac{1}{G_{4}}\right) \cosh q_{3}(l+L)-\frac{1}{G_{3}}\right]\right. \\
& \left.-\frac{1}{G_{4}}\left[\cosh q_{4} l \sinh q_{3}(l+L)+Q_{s} \cosh q_{3}(l+L) \sinh q_{4} l\right]\right\}\left(\frac{1}{\Delta_{2}}\right) .
\end{aligned}
$$

Here, the parameters are defined as

$$
\Delta_{2}=Q_{s} \cosh q_{3}(l+L) \sinh q_{4}(l+L)+\cosh q_{4}(l+L) \sinh q_{3}(l+L)
$$

and

$$
Q_{s}=\frac{k_{1} q_{3}}{k_{2} q_{4}}
$$

\section{Frequency Response}

The steady-state frequency response for the two material supported thermocouple is obtained from Eq. (36a) or (36b) by setting $x=0$ : 


$$
T(0)=\frac{1}{\Delta_{1}}\left\{Q_{1} \sinh q_{2} l\left[T_{c}+\frac{1}{G_{1}}\left(\cosh q_{1} l-1\right)\right]+\sinh q_{1}\left\{\left[T_{b}+\frac{1}{G_{2}}\left(\cosh q_{2} l-1\right)\right]\right\}\right.
$$

where $T_{a}$ and $T_{b}$ are given by Eqs. (38) and (39).

It should be noted that the steady-state frequency response Eq. (40) provides a wire temperature that is continuous everywhere and conserves the heat flux at the junction $x=0$. This represents an approximate solution since the heat flux at the interface between the large and small wires $x= \pm l$ has been neglected.

\section{RESULTS}

The amplitude ratio and phase angle of the thermocouple frequency response were plotted graphically for the case of a one material wire as shown in the schematic of fig. 1. In this case, average properties of a type B or $P t / 6 \% R h-P t / 30 \% R h$ were used since the material properties were nearly equal across the thermocouple junction. The wire dimensions, properties and gas conditions are listed in table 1(Touloukian et al., 1970).

The amplitude ratio and phase angle were also plotted for a two material thermocouple wire as shown in the schematic of fig. 2. In this case, a type B thermocouple described in table 1 was used in addition to a type $\mathrm{T}$ or copper constantan described in table 2 (Touloukian et al., 1970).

The form of the convective heat transfer coefficient $h$ that appears in the 
computation of the natural frequency $\omega_{n}$ defined in Eq. (8) was determined from the expression (Scadron and Warshawsky 1952)

$$
N u=.485 \operatorname{Re}^{1 / 2} \operatorname{Pr}^{1 / 3}
$$

where $N u\left(=h D / k_{f}\right)$ is the Nusselt number, $k_{f}$ is the thermal conductivity of the ambient fluid, $\operatorname{Pr}\left(=v_{f} / \alpha\right)$ is the Prandl number, and $\operatorname{Re}\left(=v D / v_{f}\right)$ is the Reynolds number of the thermocouple wire. Here, $\mathrm{v}$ and $v_{f}$ are the fluid velocity and kinematic viscosity, respectively. It should be noted that the convective heat transfer coefficient $h \alpha D^{1 / 2}$ and the natural frequency of a thermocouple wire for given material properties $\omega_{n} \alpha D^{-3 / 2}$.

1. One Material Thermocouple

The amplitude ratio $|T(0)|$ at the wire junction for the steady-state frequency response derived from Eqs. (17) and (32) is shown in fig. 3. This assumes a type B thermocouple wire with the dimensions listed in table 1 . In this case, the average material properties listed in table 1 were used since the one material theory assumes that the properties of the thermocouple wire are uniform across the junction. It is evident in fig. 3 that the amplitude ratio derived from the approximate expression Eq. (17) is nearly identical to the exact derivation Eq. (32). Thus, it appears that the conservation of heat flux at the interface between the small and large wires of the schematic of fig. 1 is of secondary importance.

Also included in fig. (3) are the numerical computations of Stocks (1986). 
These solutions represent explicit finite difference solutions to the one dimensional unsteady heat transfer equation as shown in Eq. (1). The small deviation of the numerical results from the exact solution at low frequency in fig. (3) is apparently due to the unsteady character of the numerical results. Similar computations of the phase angle $\Phi$ for the type B thermocouple are represented in fig. 4. As indicated, the phase angle varies over the range $0 \geq \Phi \geq-\pi / 2$ and approaches the lower limit of $-\pi / 2$ for large frequencies $\omega / \omega_{n} \gg 1$.

The spacial variation of the amplitude ratio $|T(x)|$ derived from Eqs. (17) and (32) is graphed in fig. 5. These computations were made at an angular frequency of $\omega / \omega_{n}=0.1$ for the type $B$ thermocouple. As evident in fig. 5 , the difference between the exact and approximate expression is somewhat exaggerated at a very low frequency. Nevertheless, the error represented by the approximate solution is less than $7 \%$ over the length of the thermocouple. As stated earlier, matching the heat flux at the interface between the small and large wire at $x= \pm l$ in the schematic of fig. 1 appears to be of secondary importance in relation to providing a continuous temperature profile along the wire.

The amplitude ratio $|T(0)|$, phase angle $\Phi$ and spacial variation $|T(x)|$ are also plotted in figs. (6), (7) and (8), respectively, from the steady-state frequency response represented by Eqs. (17) and (32). These results represent a type B thermocouple with a smaller diameter ratio $D_{2} / D_{1}=2$ (see table 1 for dimensions). As indicated in figs. (6), (7) and (8), the approximate and exact solutions represent comparable results in all three cases. Thus, the diameter ratio of the large and small wire 
appears to have little effect in the favorable comparison between the approximate and exact solutions representing the one material steady-state frequency response.

\section{Two Material Thermocouple}

The amplitude ratio $|T(0)|$ at the wire junction for the steady-state frequency response derived from Eqs. (17) and (40) is shown in fig. 9. This assumes a type B thermocouple wire with the dimensions listed in table 2 . In this case, the average material properties listed in Table 2 were used for the amplitude of the frequency response derived from the one material solution of Eq. (17). Also plotted in fig. 9 is the amplitude ratio derived from the two material solution of Eq. (40). In the latter case, the individual material properties listed in Table 2 were used.

As expected, the amplitude ratio for the steady-state frequency response of a type $B$ thermocouple is nearly identical with either the one material or two material approximate solutions. This is a consequence of roughly equal material properties across the junction for type $B$ thermocouples. This plot also validates the two material approximate solution Eq. (40) and the values of the boundary conditions for $T_{a}$ and $T_{b}$ substituted from Eqs (38) and (39). The same conclusion can be drawn with respect to the phase angle $\Phi$, shown in fig. 10 .

The spacial variation of the amplitude ratio $|T(x)|$ derived from Eqs. (17) and (40) is plotted in fig. 11. These computations were made at an angular frequency of $\omega / \omega_{n}=0.1$ for the type $B$ thermocouple with the dimensions listed in table 2 . It is 
interesting to note the small asymmetry in the amplitude ratio on the left and right side of the junction. This asymmetry is the result of the small differences in the physical properties across the junction as listed in table 2.

The steady state amplitude ratio $|T(0)|$ for a type $\mathrm{T}$ thermocouple is plotted in fig. 12 using equations (17) and (40). The dimensions and material properties are listed in table 2. The average material properties listed in Table 2 were used to compute the amplitude ratio of the frequency response with the one material solution, Eq. (17). Also plotted in fig. 12 is the amplitude ratio derived from the two material solution, Eq. (40). In the latter case, the individual material properties also listed in table 2 were used.

As indicated in fig. 12, the amplitude ratio for the approximate one material steady-state frequency response of a type $T$ thermocouple is distinctly different from the two material approximate solution. This is a consequence of unequal material properties across the junction for the type $T$ thermocouple. Also shown in fig. 12 is a numerical solution of the second order ordinary differential equation for the temperature, Eq. (6). The numerical finite difference solution of the boundary value problem of Eq. (6) matches both the temperature and heat flux at $x$ $=0$ and $x= \pm l$ in the schematic of fig. 2. It is clear from fig. 12 that the approximate two material analytical solution, equation (40), is in good agreement with the numerical results despite some differences at low frequencies $\omega / \omega_{1}<.04$. Similar results are indicated for the phase angle $\Phi$ in fig. 13.

The spacial variation of the amplitude ratio $|T(x)|$ derived from Eqs. (17) and 
(40) is graphed in fig. 14. These computations were made at an angular frequency of $\omega / \omega_{1}=0.1$ for the type $T$ thermocouple. As evident in fig. 14, the two material solution derived from Eq. (40) accurately represents the features of the asymmetry associated with a type $\mathrm{T}$ thermocouple. In particular, the relatively large resistance to axial heat conduction in the constantan wire on the right of the junction is

reflected in the larger values of the amplitude ratio $|T(x)|$. Also shown in fig. 14 is the approximate one material solution represented by Eq. (17). In the latter case, the average values for the material properties of a type $T$ thermocouple were used as listed in Table 2. Therefore, one can conclude that for thermocouples whose material properties on either side of the junction are markedly different, the two material solution developed in this paper is a substantial improvement in accuracy both in frequency response and in temperature distribution along the wire.

\section{Experimental Procedure}

In the experiment described below, thermocouple sensors were exposed to a constant velocity air stream of varying temperature. In particular, the dynamic response of the thermocouple sensors to a square wave temperature profile was measured for a range of frequencies.

\section{Rotating Wheel Experiment}

A rotating wheel configuration was used to deliver the test airstream to the proposed sensors. A similar experimental apparatus is described in detail by 
Elmore et al. (1986). A schematic of the rotating wheel apparatus used in the present experiment is shown in figure 15. As the wheel rotates, the holes pass the two air supply tubes which allow slugs of hot and cold air to alternately enter a transition tube assembly mounted directly above the rotating wheel. In the transition tube the slugs of hot and cold air coalesce into a single air stream providing roughly a square wave temperature profile covering a range of frequencies from roughly 1 to $30 \mathrm{~Hz}$.

The analog temperature signal was digitized with a Data Translation DT-2801 board mounted in an expansion slot of an IBM AT compatible computer as shown in figure 15. The ASYST software loaded on the hard disc of the personal computer provided a flexible system for data storage, manipulation and display.

The true temperature profile of the airstream was measured with a constant current anemometer (TSI 1054-A) and sensor (1226 PI 2.5).

\section{Thermocouple Construction}

Thermocouple wire of the desired length and type is threaded into the four hole ceramic with the thermocouple end last. Three or four kinks are made in each wire near the thermocouple end so the wire must be firmly pulled into the tube leaving enough wire sticking out to make the thermocouple. Drops of epoxy are picked up with a piece of .010" diameter wire, added to the ceramic tube at $\mathrm{A}$ (see fig. 16) and pushed down around both wires. The kinks and epoxy firmly fasten the wires in the ceramic so they do not twist when the free ends are 
manipulated for electrical connections.

The junction at B in figure 16 is made by cutting the large wire about half way through with a razor blade, laying the small diameter wire in the cut, and welding the cut closed with a stored energy spot welder. After the weld, the excess wire ends that protrude through the weld area are bent and broken off to clean up the junction.

For the chromel alumel couples, the junction at $C$ of figure 16 was made using a stored energy spot welder. The wires are crossed and welded and then trimmed with a razor blade and bent with tweezers under a microscope until collinear.

A stored energy spot welder will not work for the copper constantan couples. For these couples the junctions were made by silver soldering. The silver solder wire is coated with flux and the end heated with a torch until a drop forms. A twisted pair of thermocouple wires is pushed briefly into the flux coated solder drop. The solder will wet the pair up to where the twist stops. Again using a razor blade and tweezers under a microscope, the thermocouple is bent and trimmed until collinear.

\section{ASYST Software}

ASYST software was developed to acquire temperature data simultaneously from the thermocouple and constant current anemometer. Data were digitized for two channels at a sampling rate of $512 \mathrm{~Hz}$ per channel for a total sample time of 
two seconds. The ASYST software code is listed in figure 17.

The ASYST code first plotted the temperature profiles versus time from the thermocouple and anemometer and an example of the plot is shown in figure 18. The ASYST software next took the Fast Fourier Transform of the temperature data in each channel and recorded the amplitude ratio and phase angle between both channels at the first harmonic for the square wave. These data are discussed in the next section.

VI. Results

Initial tests with a signal generator and the output from an RC circuit indicated that the ASYST code and data acquisition hardware were operating properly. Several test sensors were constructed and tested with the dimensions listed in table 3. In each case measurements of the amplitude and phase angle were compared with the appropriate theory. A discussion of the results is given below.

\section{First Order Response}

The amplitude ratio and phase angle were measured with the type $K$ thermocouple listed first in table 3. The lengths of the thermocouple and support wires in this case were chosen to eliminate the effects of axial heat conduction. The experimental data representing the amplitude ratio are plotted in figure 19 along with the theory representing a first order frequency response. The experimental data were correlated with a natural frequency $\omega_{1}=5.5 \mathrm{sec}^{-1}$ and a 
thermal diffusivity of $\alpha=.059 \mathrm{~cm}^{2} / \mathrm{sec}$.

As indicated in figure 19 the data has been correlated with the theory to within $10 \%$. Although the experimental data is reproducible, there is a noticeable drift of the data relative to the theory with increasing wheel frequency $\omega$. The drift is probably due to a small increase in the natural frequency $\omega_{1}$ of the thermocouple wire with increasing wheel frequency $\omega$.

The natural frequency $\omega_{1}$ of Equation (10) depends on accurate predictions of the convective heat transfer coefficient $h$ from Equation (41). However, there may be a small change in either the mean air velocity or unsteadiness in the mean velocity which would lead to small changes in the heat transfer coefficient $h$ and the natural frequency $\omega_{1}$.

Also plotted in figure 20 are experimental data representing the phase angle $\Phi$ for the first order frequency response of the type $\mathrm{K}$ thermocouple. In this case the data is correlated to within $7 \%$ of the theory. The experimental data for the phase angle also indicate a small drift in the natural frequency $\omega_{1}$ as was apparent in figure 19.

\section{Frequency Response (one material)}

The amplitude ratio and phase angle were measured with the second type $\mathrm{K}$ thermocouple listed in table 3. The lengths of the thermocouple and support wires in this case were chosen (i.e., shortened) to demonstrate the effects of axial 
heat conduction. The experimental data representing the amplitude ratio are plotted in figure 21 along with the theoretical prediction given by Eq. 32 . Both the data and theory were correlated by assuming that the material properties are uniform across the thermocouple junction. In this case the data were correlated with a natural frequency $\omega_{1}=5.0 \mathrm{sec}^{-1}$ and a thermal diffusivity of $\alpha=.059 \mathrm{~cm}^{2} / \mathrm{sec}$.

As indicated in figure 21 the data has been correlated with the theory to within $10 \%$ over most of the range of wheel frequencies. The data is reproducible but again a noticeable drift of the experimental data exists relative to the theory as was discussed in the previous section.

Also plotted in figure 22 are experimental data representing the phase angle $\Phi$ for the frequency response of the shortened type $\mathrm{K}$ thermocouple. The data is correlated to within $7 \%$ of the theory. The experimental data for the phase angle also indicate a small drift in the natural frequency $\omega_{1}$. 
IV. NOMENCLATURE
A $=$ constant of integration
B $=$ constant of integration
c $\quad=\quad$ material specific heat $\left(J-g m^{-1}-{ }^{0} K^{-1}\right)$
D $=$ thermocouple wire diameter $(\mathrm{cm})$
G $\quad=1+i\left(\omega / \omega_{n}\right)$
h $=$ heat transfer coefficient $\left(J-c m^{-2}-s^{-1}-{ }^{0} K^{-1}\right)$
i $\quad=\quad$ unit imaginary number $(=\sqrt{-1})$
$\mathrm{k}=$ material thermoconductivity $\left(J-c m^{-1}-s^{-1}-{ }^{0} K^{-1}\right)$
$\mathrm{k}_{\mathrm{f}}=$ gas thermoconductivity $\left(J-c \mathrm{~m}^{-1}-\mathrm{s}^{-1}-{ }^{0} \mathrm{~K}^{-1}\right)$
$l=$ length of small thermocouple wire $(\mathrm{cm})$
L = length of large thermocouple wire $(\mathrm{cm})$
$\mathrm{Nu}=$ Nusselt number $\left(=h D / k_{f}\right)$
$\operatorname{Pr} \quad=\quad$ Prandl number $\left(=v_{f} / \alpha\right)$
$\mathrm{Q} \quad=\quad \frac{D_{1}^{2} q_{1}}{D_{2}^{2} q_{2}}$
$\mathrm{Q}_{\mathrm{t}} \quad=\frac{k_{1} q_{1}}{k_{2} q_{2}}$
$\mathrm{Q}_{\mathrm{s}} \quad=\frac{k_{1} q_{3}}{k_{2} q_{4}}$
$\mathrm{q}=(G / \gamma)^{-5}$
$\operatorname{Re} \quad=\quad$ Reynolds number $\left(=\mathrm{vD} / v_{\mathrm{f}}\right)$
$\mathrm{t}=$ time $(\mathrm{s})$ 


$\begin{array}{ll}\mathrm{T} & =\text { steady-state frequency response } \\ \mathrm{T}_{\mathrm{f}} & =\text { amplitude of periodic gas temperature }\left({ }^{\circ} \mathrm{K}\right) \\ \mathrm{T}_{\mathrm{g}} & =\text { gas temperature }\left({ }^{\circ} \mathrm{K}\right) \\ \mathrm{T}_{0} & =\text { mean gas temperature }\left({ }^{\circ} \mathrm{K}\right) \\ \vec{T}_{w} & =\text { complex amplitude of periodic wire temperature }\left({ }^{\circ} \mathrm{K}\right) \\ T_{w} & =\text { local wire temperature }\left({ }^{\circ} \mathrm{K}\right) \\ \mathrm{v} & =\text { gas velocity }\left(\mathrm{cm}-\mathrm{s}^{-1}\right) \\ \mathrm{x} & =\text { axial distance from center of wire }(\mathrm{cm})\end{array}$

\section{Greek Symbols}

$\alpha=\quad \quad=\quad$ thermal diffusivity $\left(\mathrm{cm}^{2}-s^{-1}\right)$

$\gamma \quad=\alpha / \omega_{n}\left(\mathrm{~cm}^{2}\right)$

$v_{f}=$ kinematic viscosity of gas $\left(\mathrm{cm}^{2}-\mathrm{s}^{-1}\right)$

$\omega \quad=\quad$ angular frequency $\left(s^{-1}\right)$

$\omega_{n} \quad=\quad$ natural frequency of wire $(=4 h / \rho c D)\left(s^{-1}\right)$

$\Phi \quad=\quad$ phase angle

$\rho \quad=\quad$ material density $\left(\mathrm{gm} \cdot \mathrm{cm}^{-3}\right)$

$\Delta_{1} \quad=\quad$ dimensionless function

$\Delta_{2} \quad=$ dimensionless function 


\section{REFERENCES}

1. Carslaw, H. S., Introduction to the Mathematical Theory of the Conduction of Heat in Solids, Dover Publications, 2nd edition (1945).

2. Dils, R. R. and Follansbee, P. S., Wide Bandwidth Gas Temperature Measurements in Combustor and Combustor Exhaust Gases, Instrumentation in the Aerospace Industry, 21 (B. Washburn, ed.), ISA 76245 (1976).

3. Elmore, D. L., Robinson, W. W. and Watkins, W. B., Dynamic Gas Temperature Measurement System: Final Report, NASA CR-168267 (1983).

4. Elmore, D.L., Robinson, W. W. and Watkins, W. B., Further Development of the Dynamic Gas Temperature Measurement System: Vol. I - Technical Efforts, NASA CR-179513 (1986).

5. Stocks, D. R., Further Development of the Dynamic Gas Temperature Measurement System: Vol. II - Computer Program User's Manual, NASA CR-179604 (1986).

6. Forney, L. J. and Fralick, G. C., Frequency Response of a Uniform Thermocouple Wire: Effects of Axial Conduction, Proceedings of the 6th Miami International Symposium on Heat and Mass Transfer (1990).

7. Fralick, G. C., Correlation of Velocity and Velocity-Density Turbulence in the Exhaust of an Atmospheric Burner, Turbine Engine Hot Section Technology - 1985, NASA CP-2465, pp. 81-85.

8. Forney, L. J. and Fralick, G. C., Frequency Response of a Thermocouple Wire: Effects of Axial Heat Conduction (submitted, International I. Heat and Mass Transfer, 1991).

9. Hildebrand, F. B., Advanced Calculus for Applications, (2nd Ed), Prentice Hall (1976).

10. Scadron, M.D. and Warshawsky, I., Experimental Determination of Time Constants and Nusselt Numbers for Bare-Wire Thermocouples in HighVelocity Air Streams and Analytic Approximation of Conduction and Radiation Errors, NACA TN-2599 (1952).

11. Touloukian, Powell, Ho and Clemens, (ed.), Thermal Physical Properties of Matter, Purdue Research Foundation, Plenum Pub. (1970). 
Table 1 - Properties of One Material Wire (Type B)

Dimensions (cm)

$\begin{array}{lcccc}\underline{D}_{1} & \underline{D}_{2} & \underline{D}_{2} / D_{1} & \underline{1} & \underline{L} \\ .025 & .05 & 2 & .2 & .35 \\ .0076^{*} & .038 & 5 & .1 & .2\end{array}$

Average Properties of Type B

Gas Properties

$\rho c\left(\frac{J}{c m^{3}-{ }^{\circ} K}\right) \quad \alpha\left(\frac{c m^{2}}{\mathrm{sec}}\right)$

$T_{0}=900^{\circ} \mathrm{K}$

3.8

.22

$M=.26$

$P=1 \mathrm{~atm}$

$* \omega_{1}=32.9 \mathrm{sec}^{-1}$ 
Dimensions $(\mathrm{cm})$

$\underline{D}_{1} \quad \underline{D}_{2} \quad \underline{D}_{2} / D_{1} \quad \underline{l} \quad \underline{L}$

$\begin{array}{llllll}.0076 & .038 & 5 & .1 & .2\end{array}$

Air Properties

$$
\begin{aligned}
& T_{0}=300^{\circ} \mathrm{K} \\
& P=1 \mathrm{~atm} \\
& V=50 \mathrm{~m} / \mathrm{sec}
\end{aligned}
$$

Properties of Type B

$$
\rho c\left[\frac{J}{c m^{3}-o k}\right] \quad \alpha\left[\frac{\mathrm{cm}^{2}}{\mathrm{sec}}\right]
$$

2.73

.238

2.86

.190

2.8

.214

\section{Properties of Type I}

$$
\rho c\left[\frac{J}{c m^{3}-{ }^{\circ} K}\right] \quad \alpha\left[\frac{\mathrm{cm}^{2}}{\mathrm{sec}}\right]
$$

Copper

Constantan

Average

Region

1

2
3.44

1.16

3.48

.067

3.46

.614

\section{Wire Location}

$\begin{array}{cll}\text { Region } & \text { Type B } & \text { Type T } \\ 1 & \mathrm{Pt}-6 \% \mathrm{Rh} & \text { Copper } \\ 2 & \mathrm{Pt}-30 \% \mathrm{Rh} & \text { Constantan }\end{array}$


Table 3 - Dimensions for Test Thermocouples (cm)

Test

First Order

Axial Conduction (one material)
Thermocouple Type

K

K
D2

.0076

.038

.0076

.038

5

$\underline{D}_{2} / D_{1} \quad 1 \quad \underline{L}$

5

$0.25 \quad 0.5$

$\begin{array}{ll}0.1 & 0.2\end{array}$ 


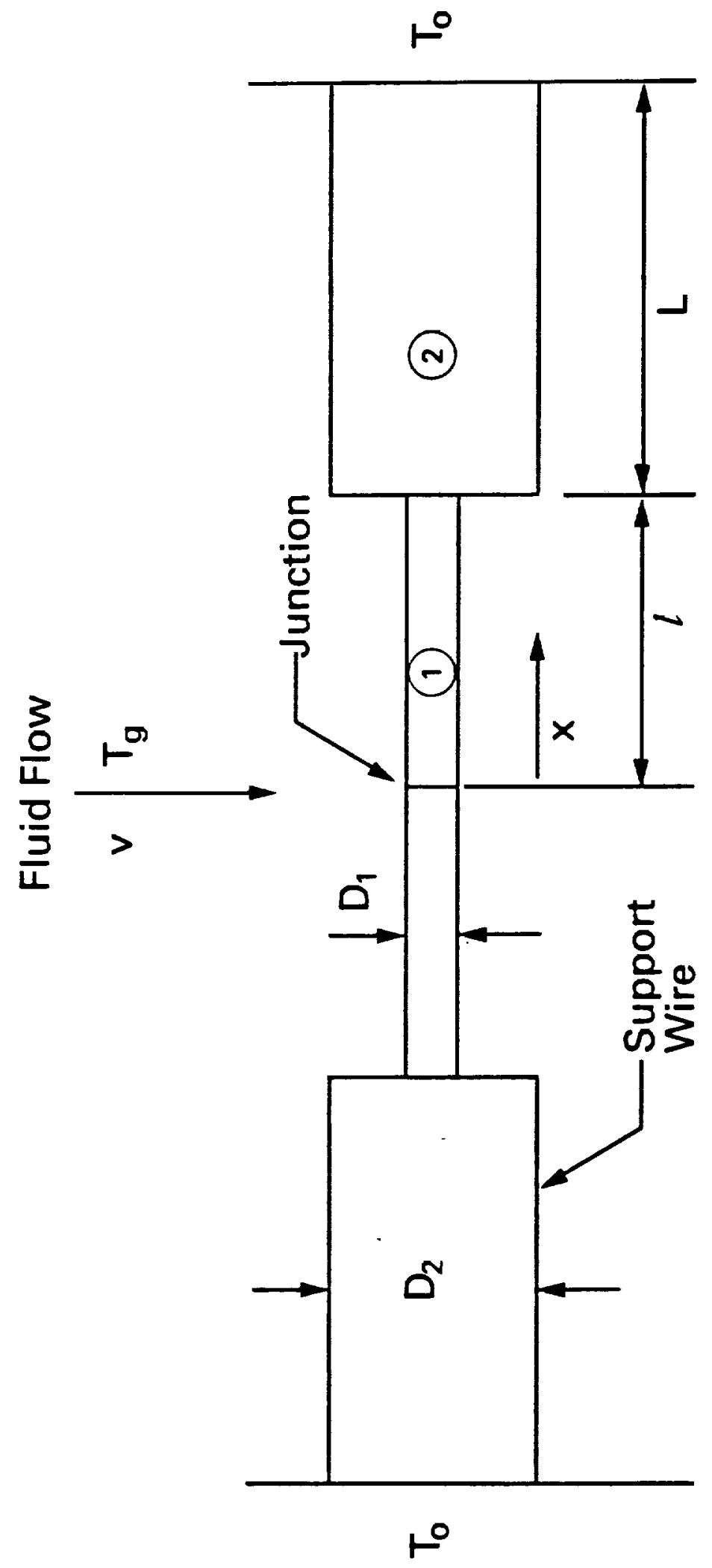

苟 


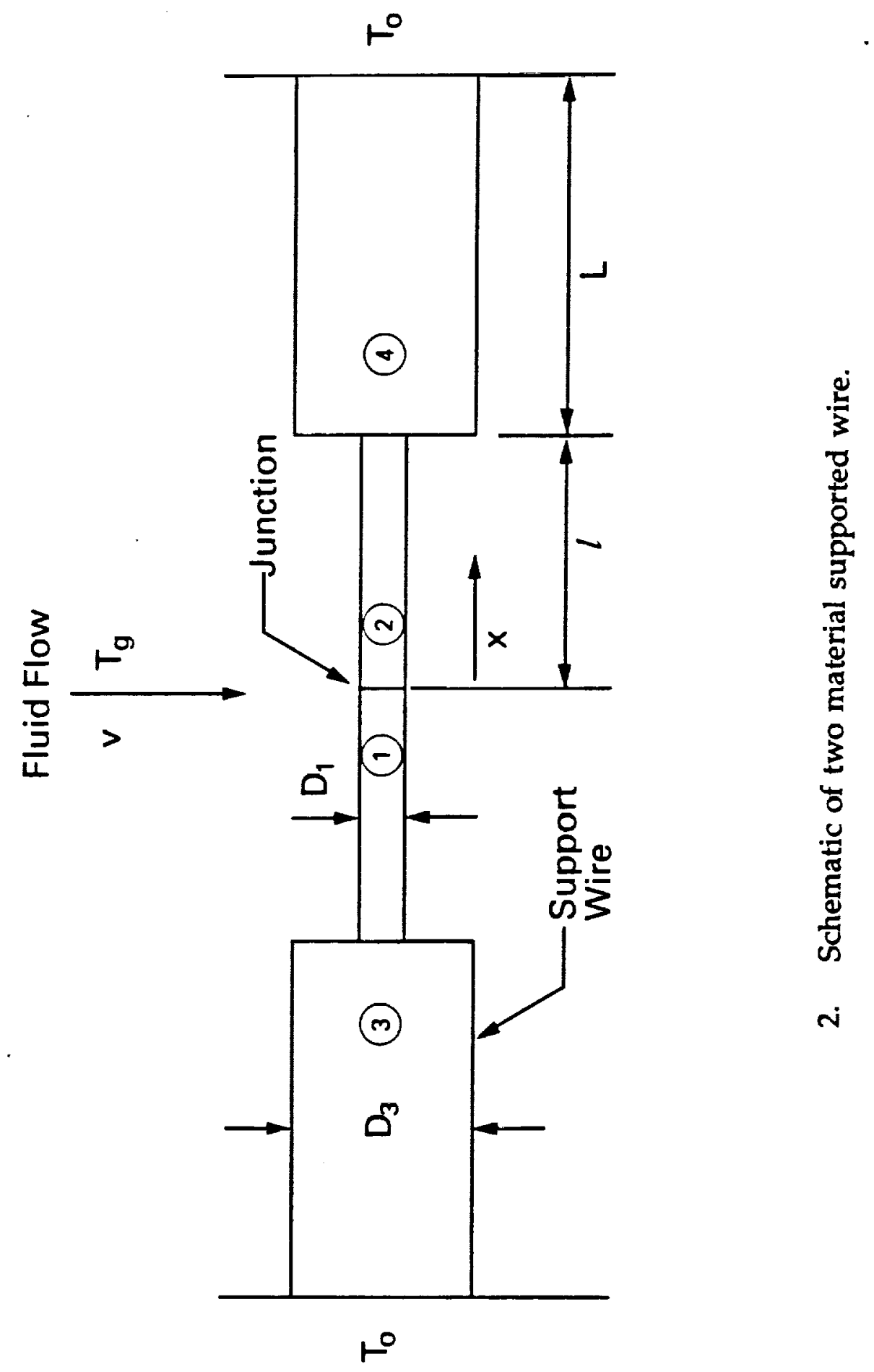




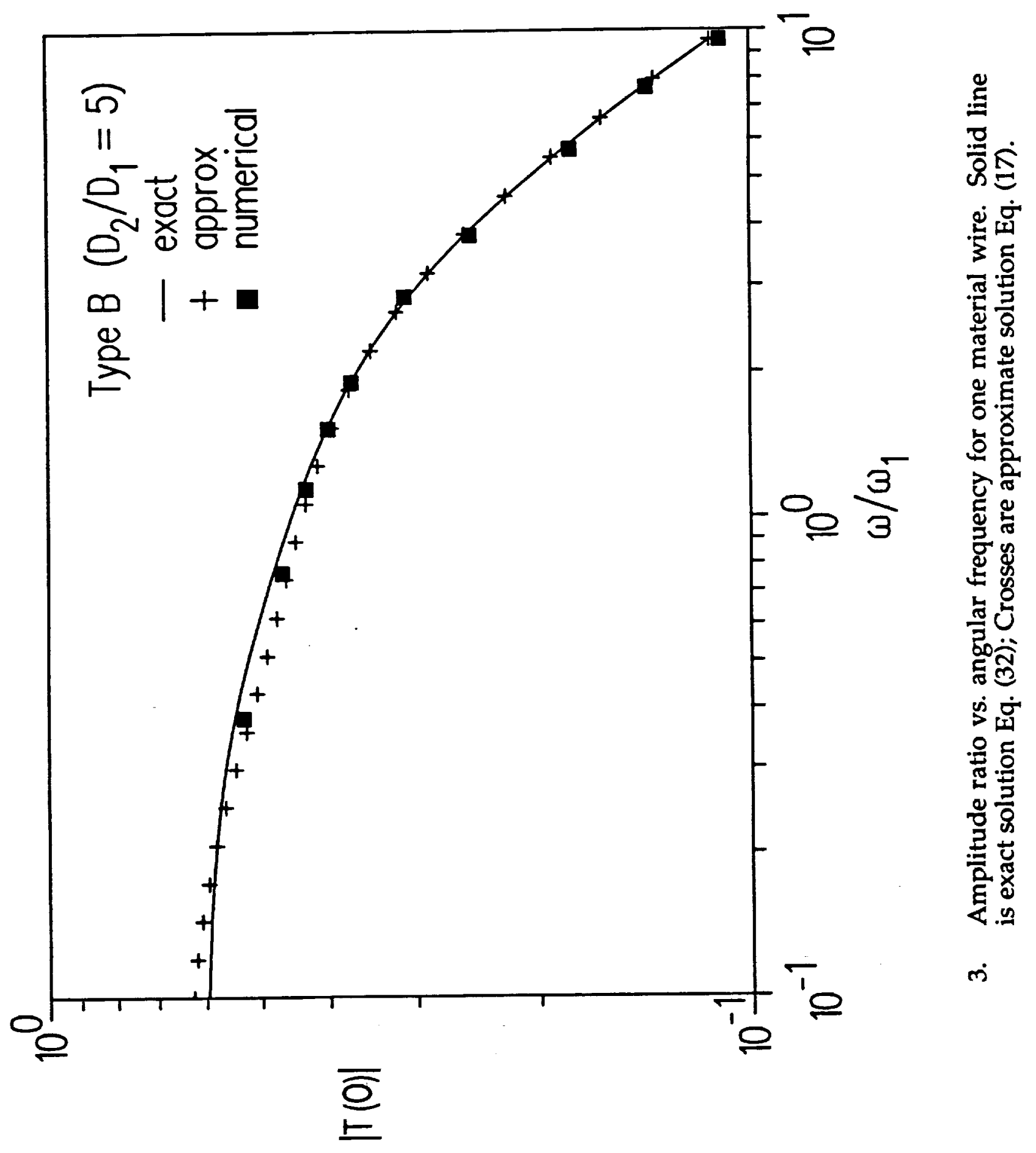




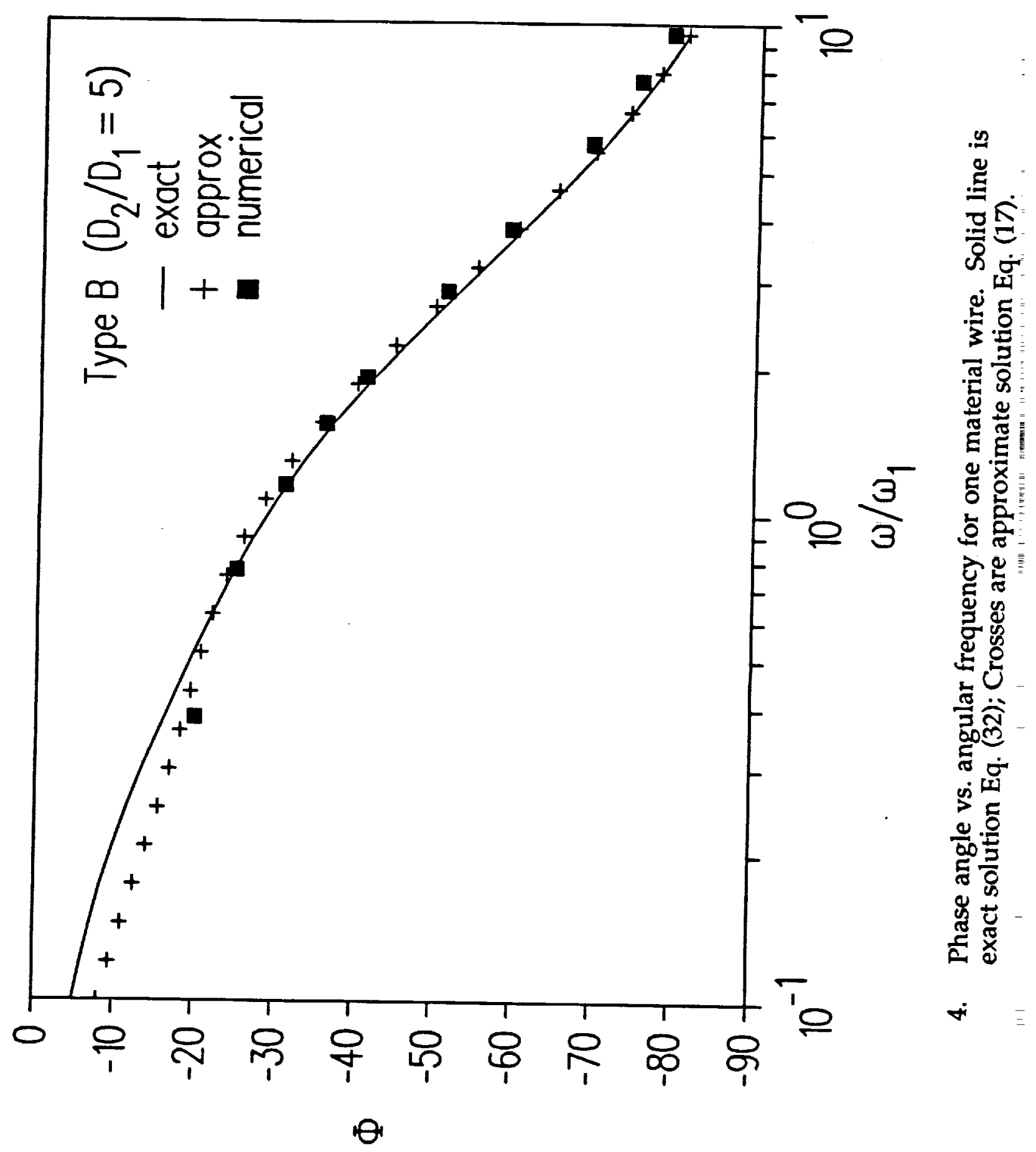




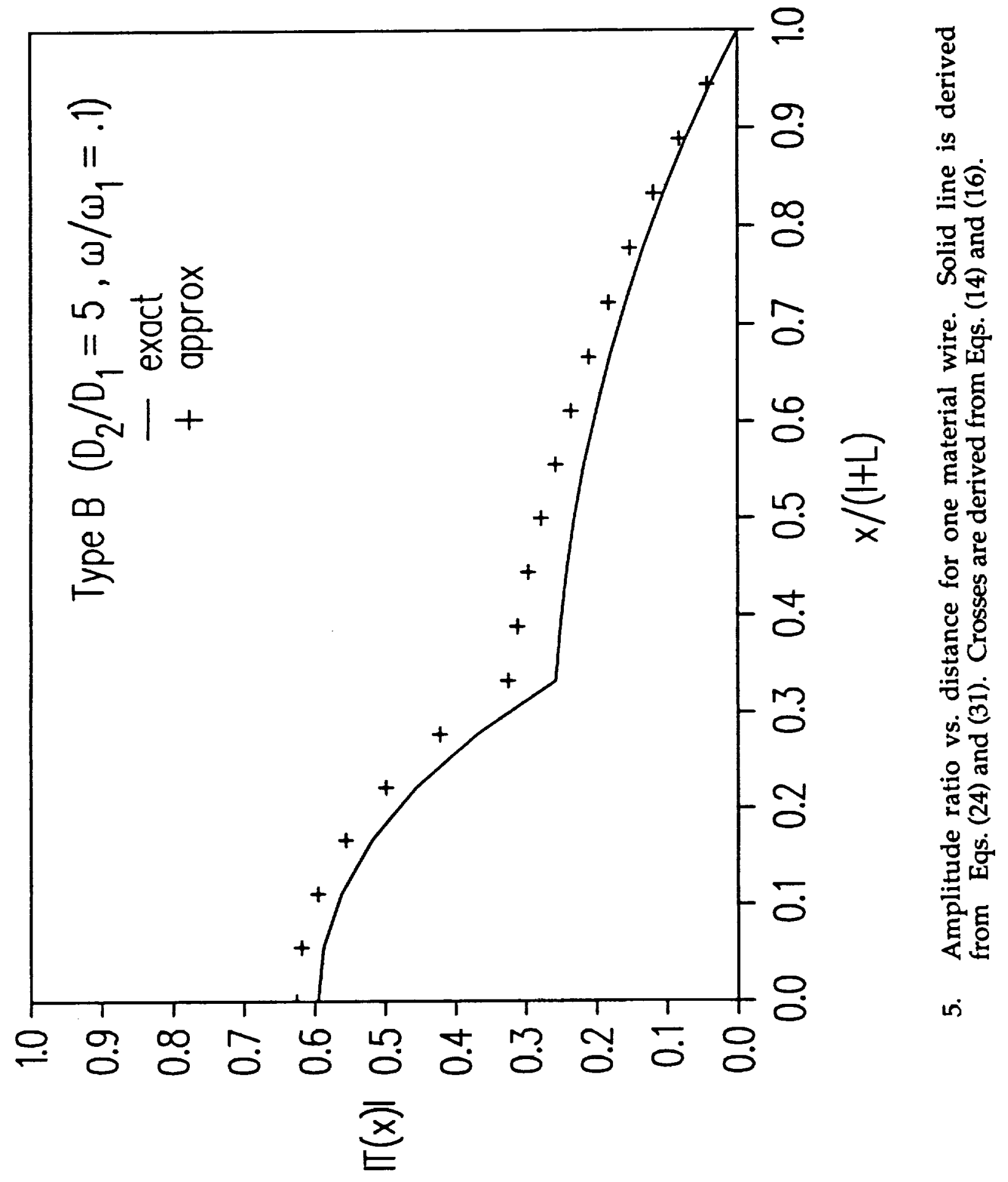




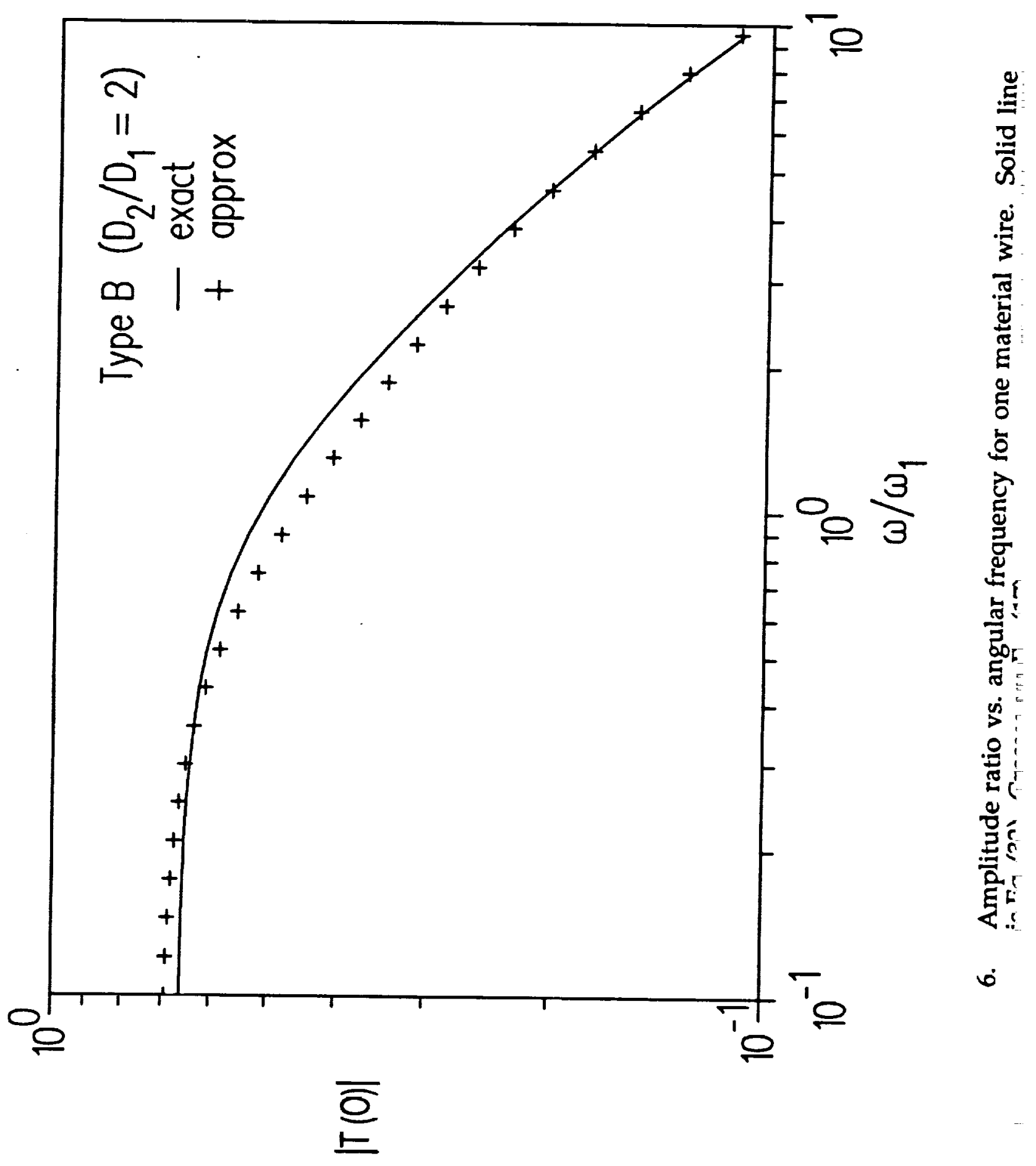




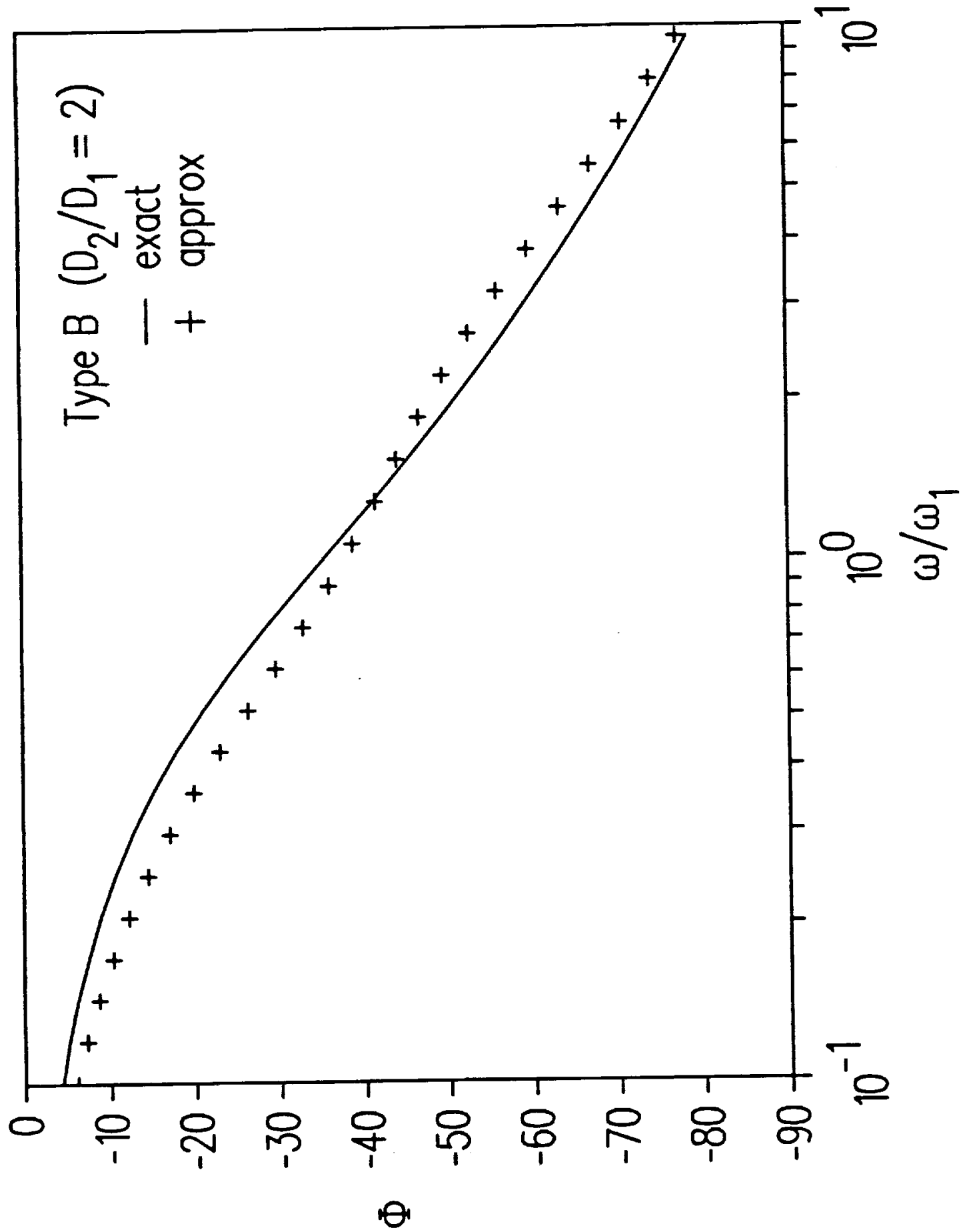

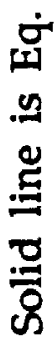

这

$\frac{\pi}{\stackrel{0}{0}}$

ปั

욤

넌

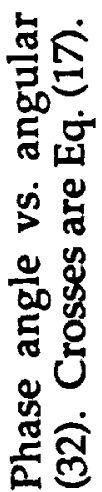

$N$ 


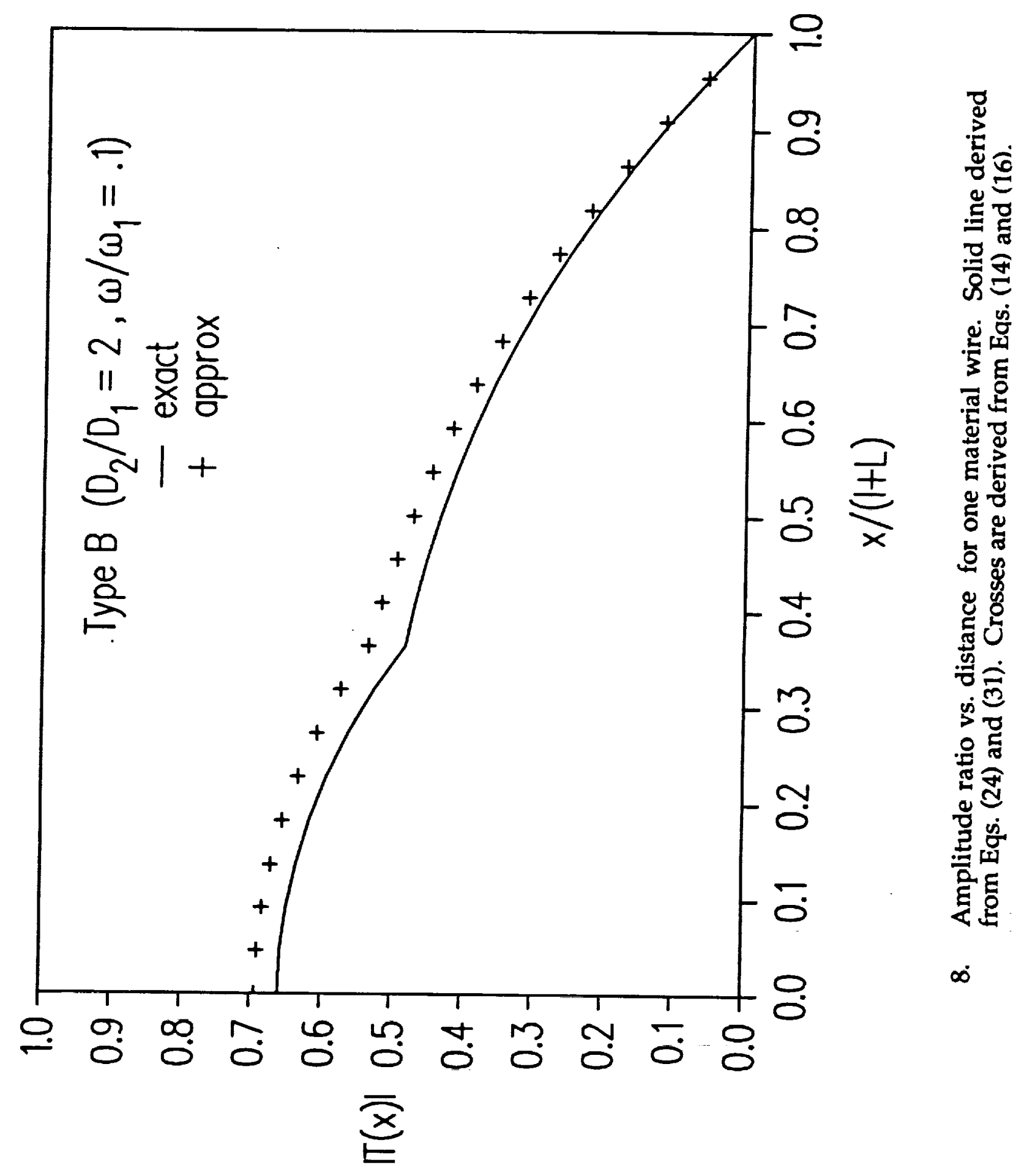




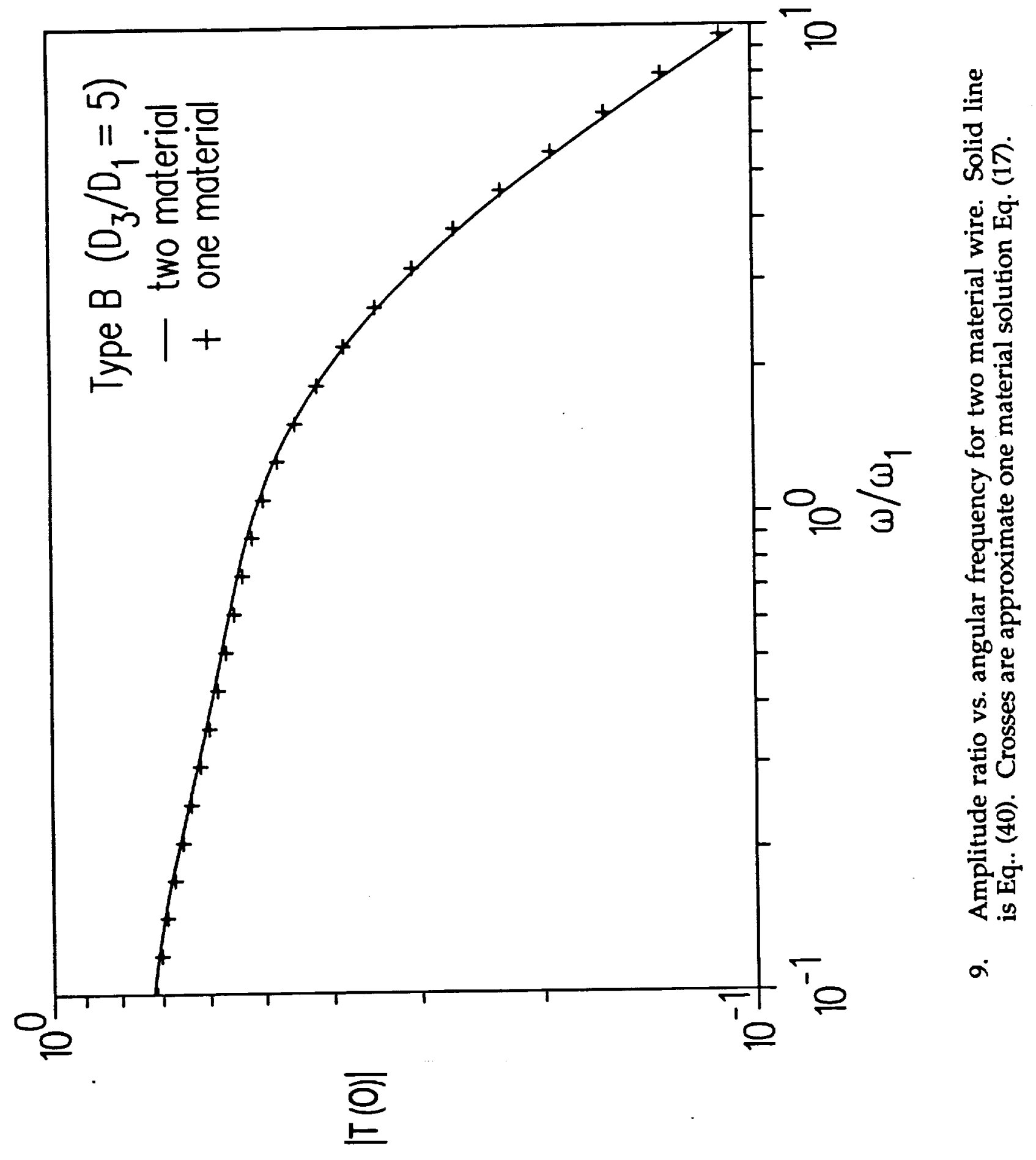




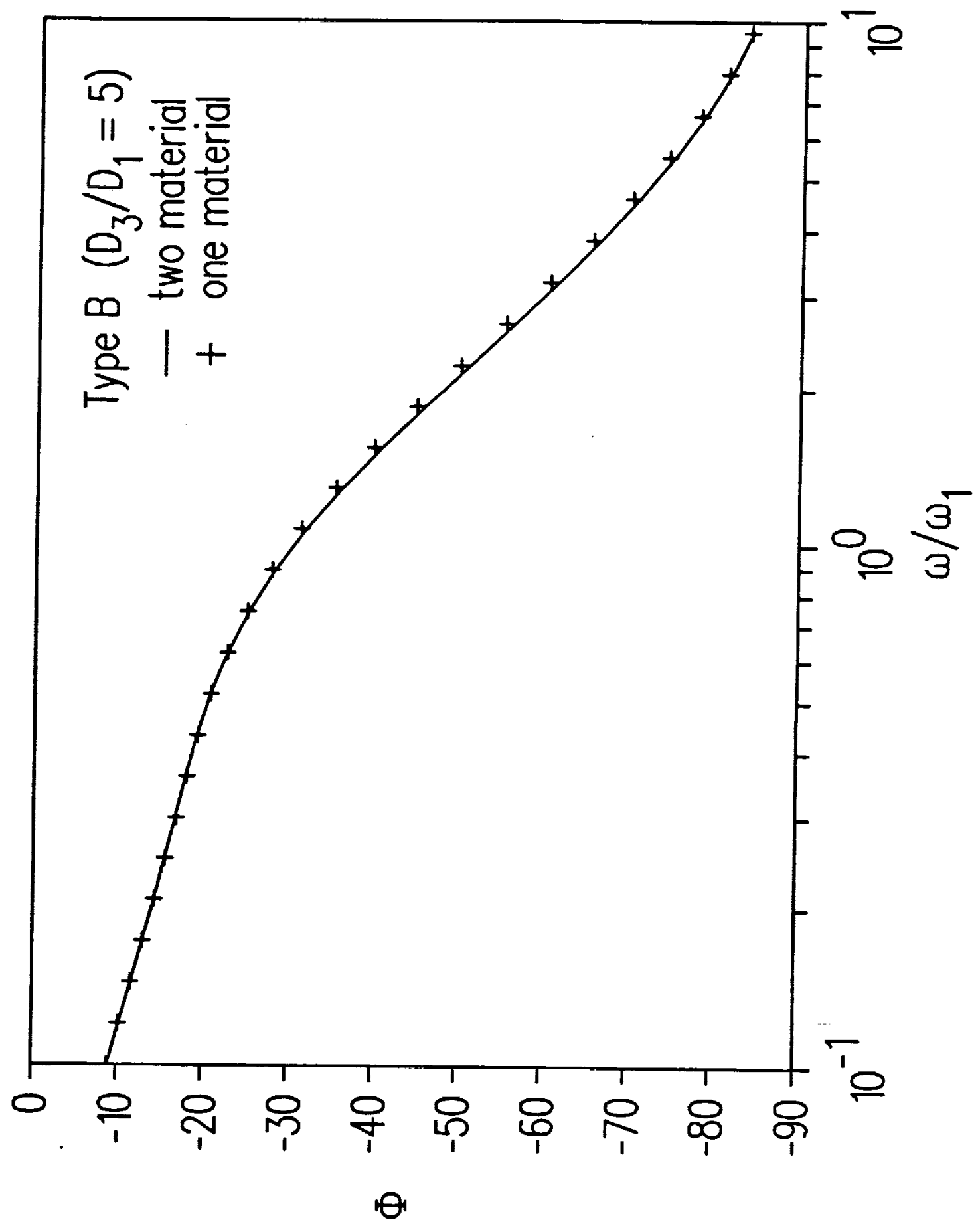

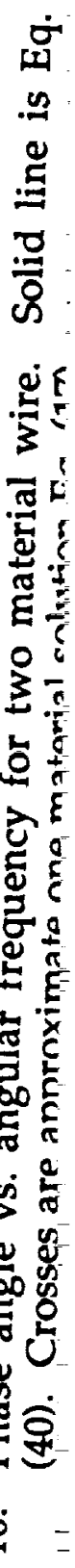




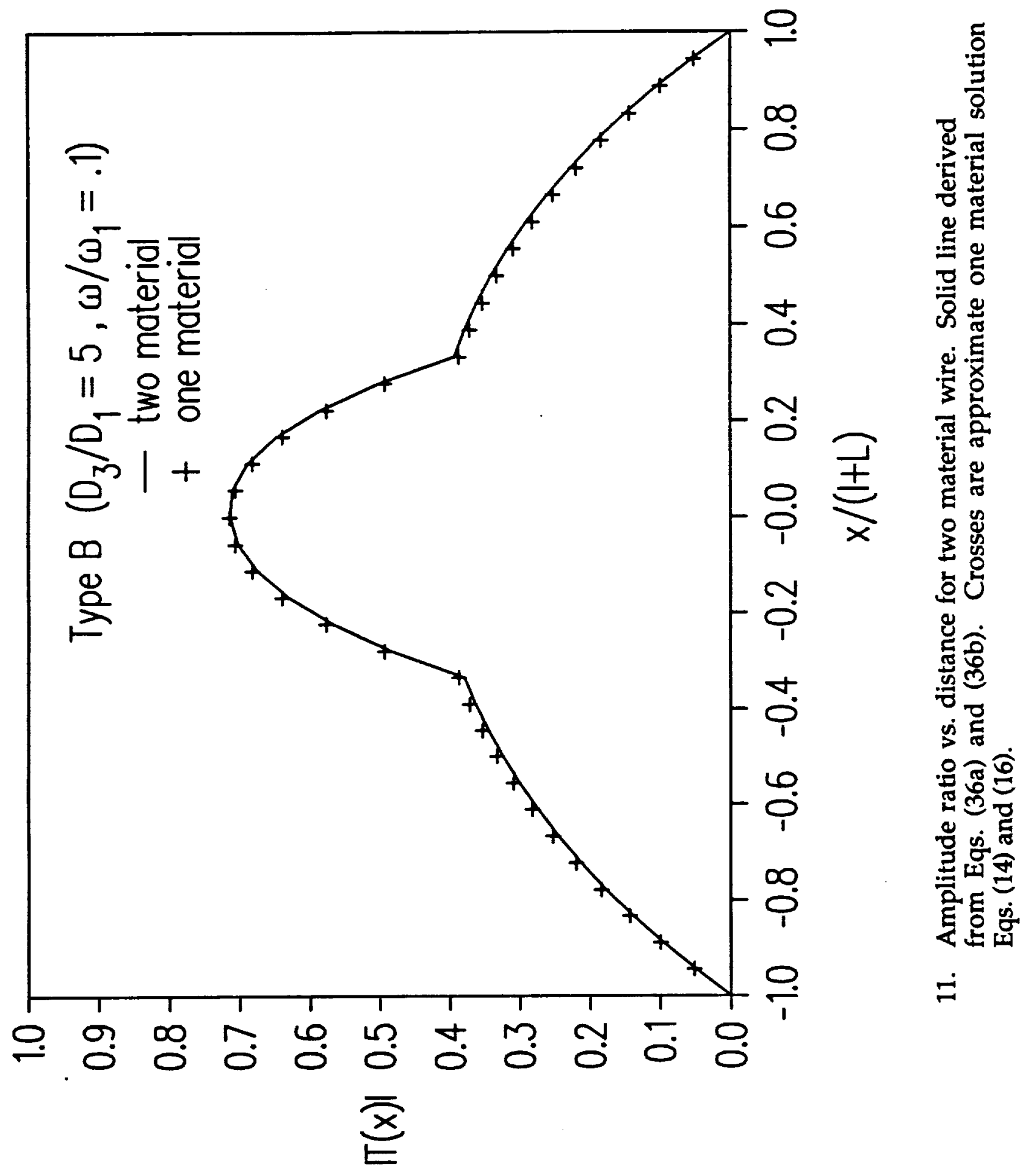




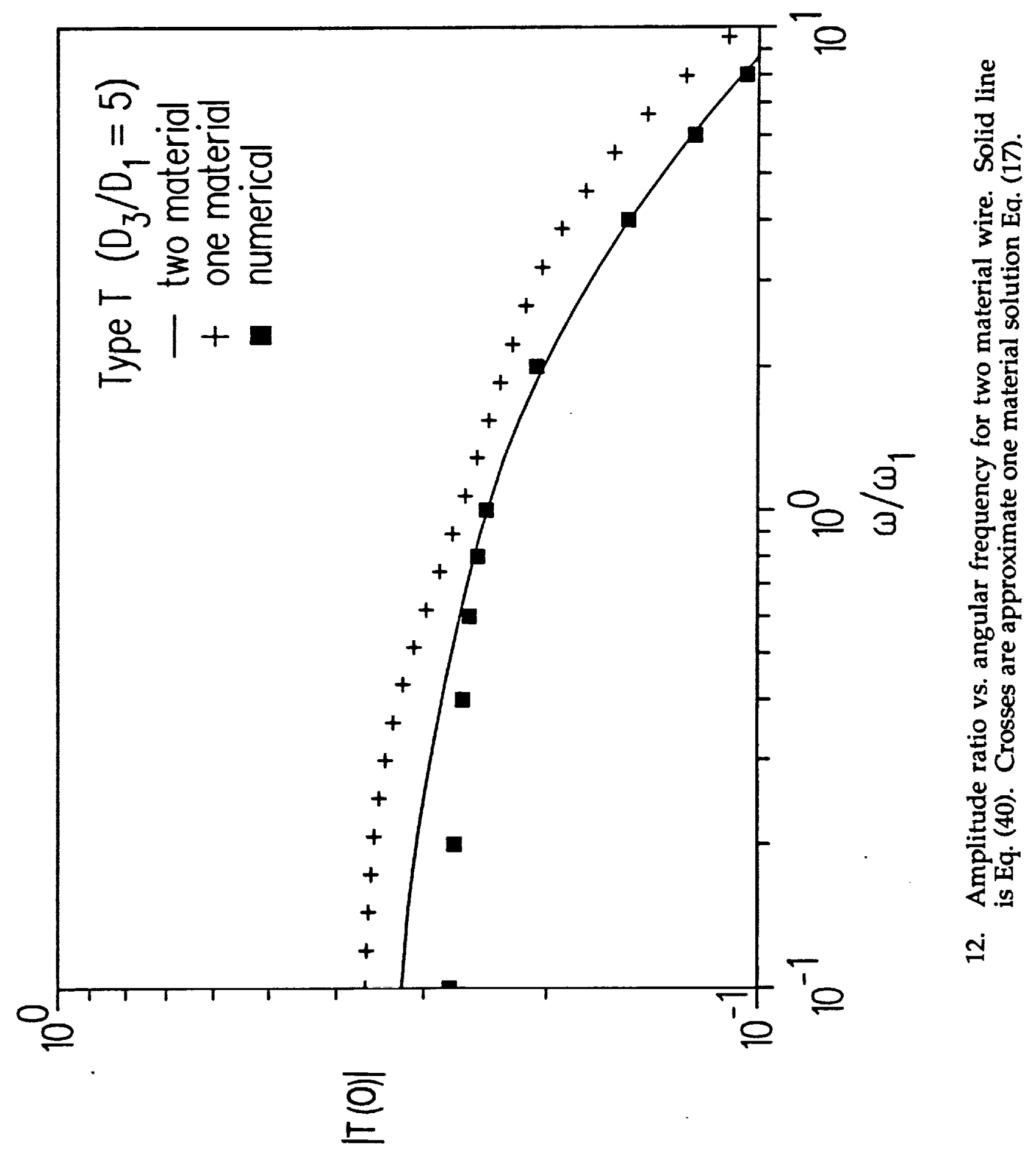




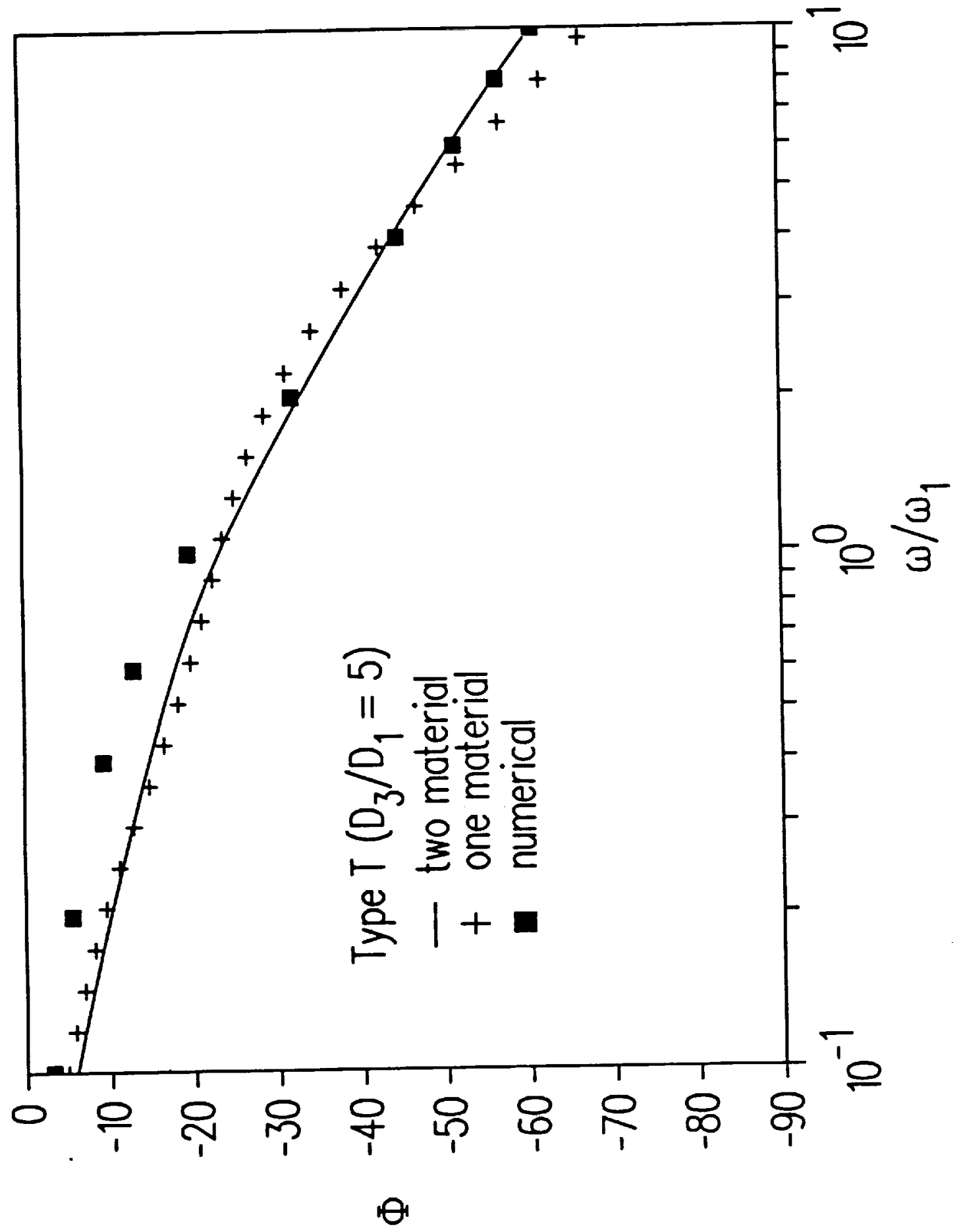

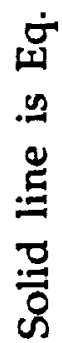

点完

논

느도

잉

열

4

글 눙

ซึ

$>$

$\triangle$

है

\&

昰

i் 


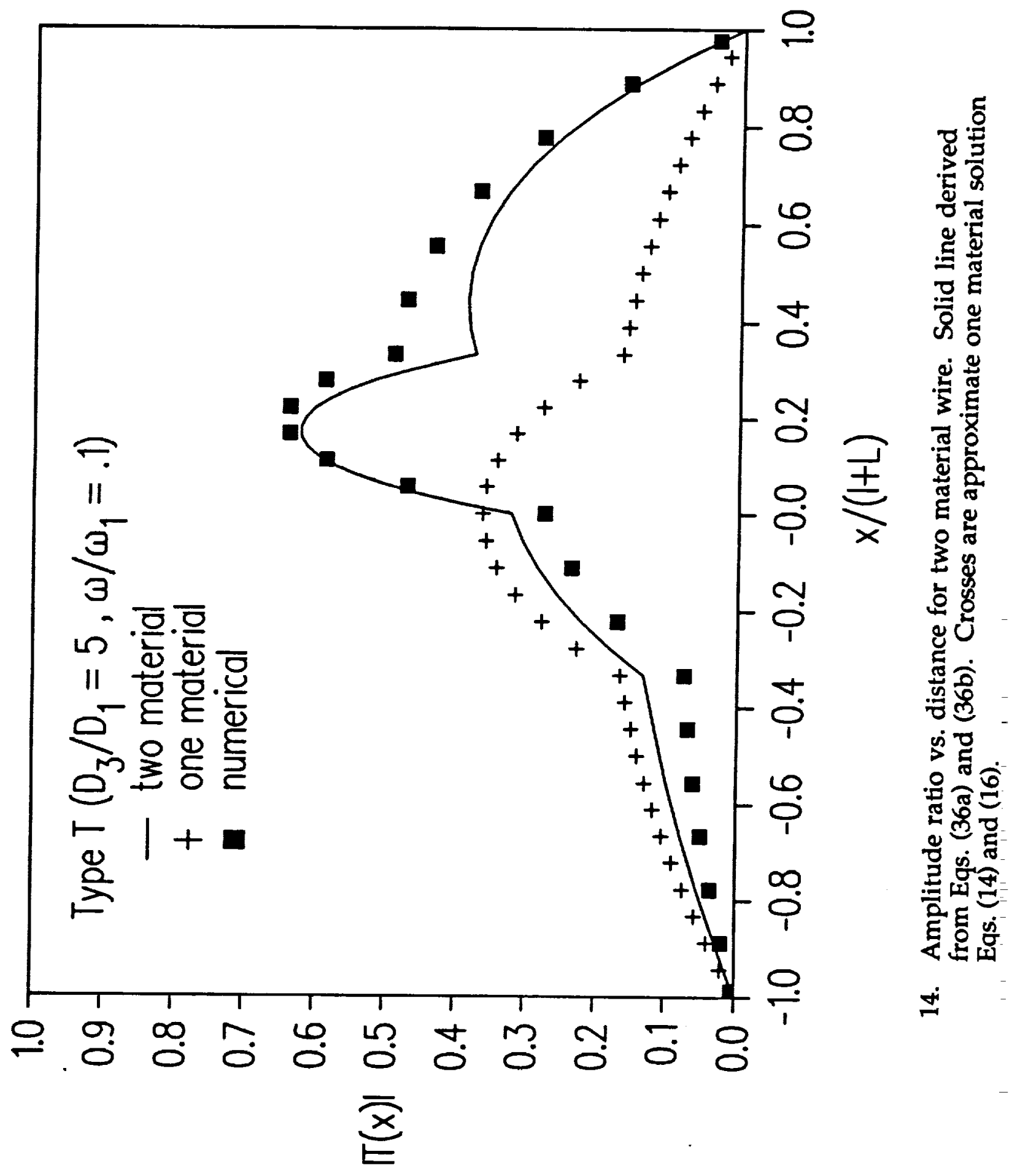




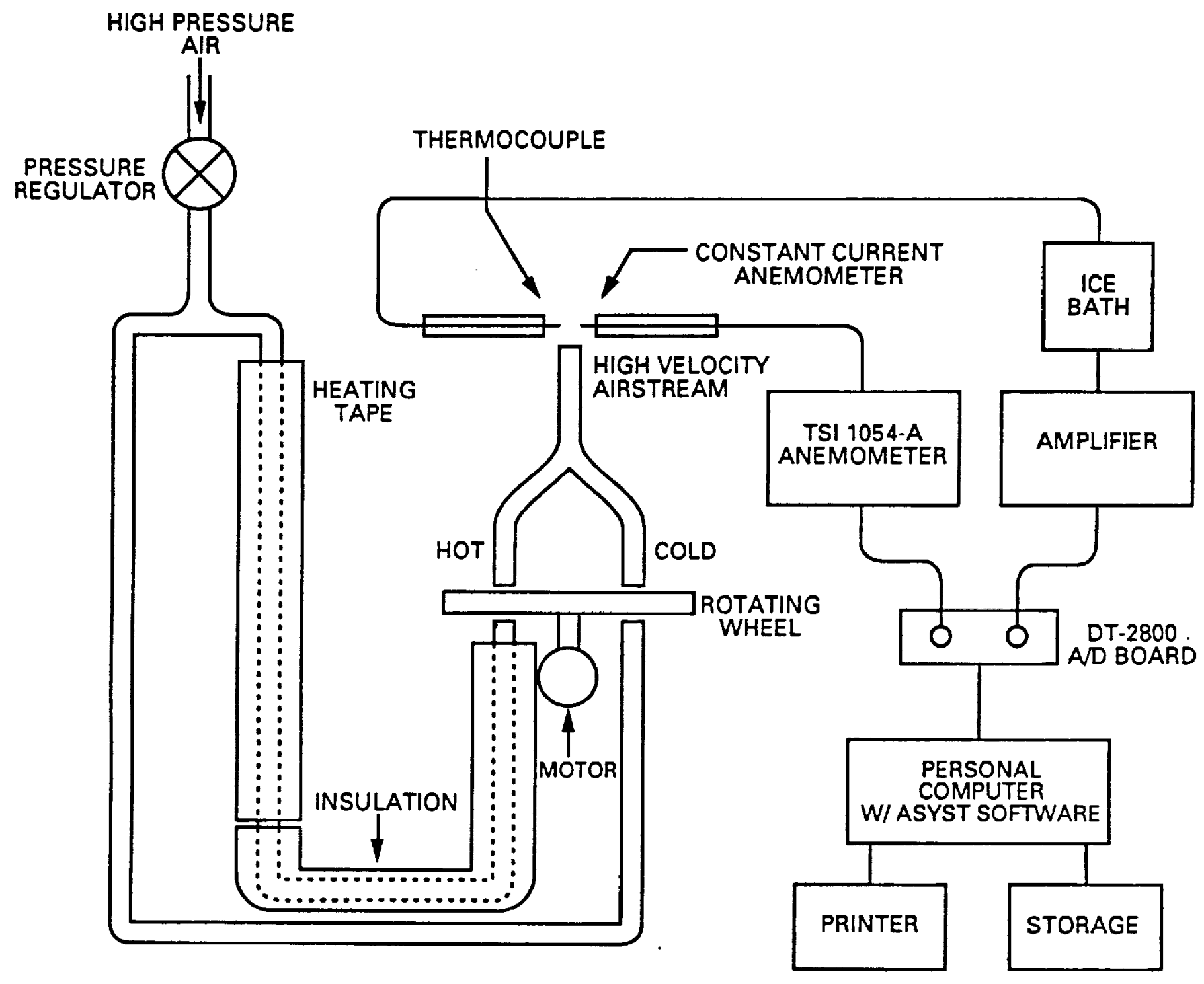

15. Rotating wheel experiment. 


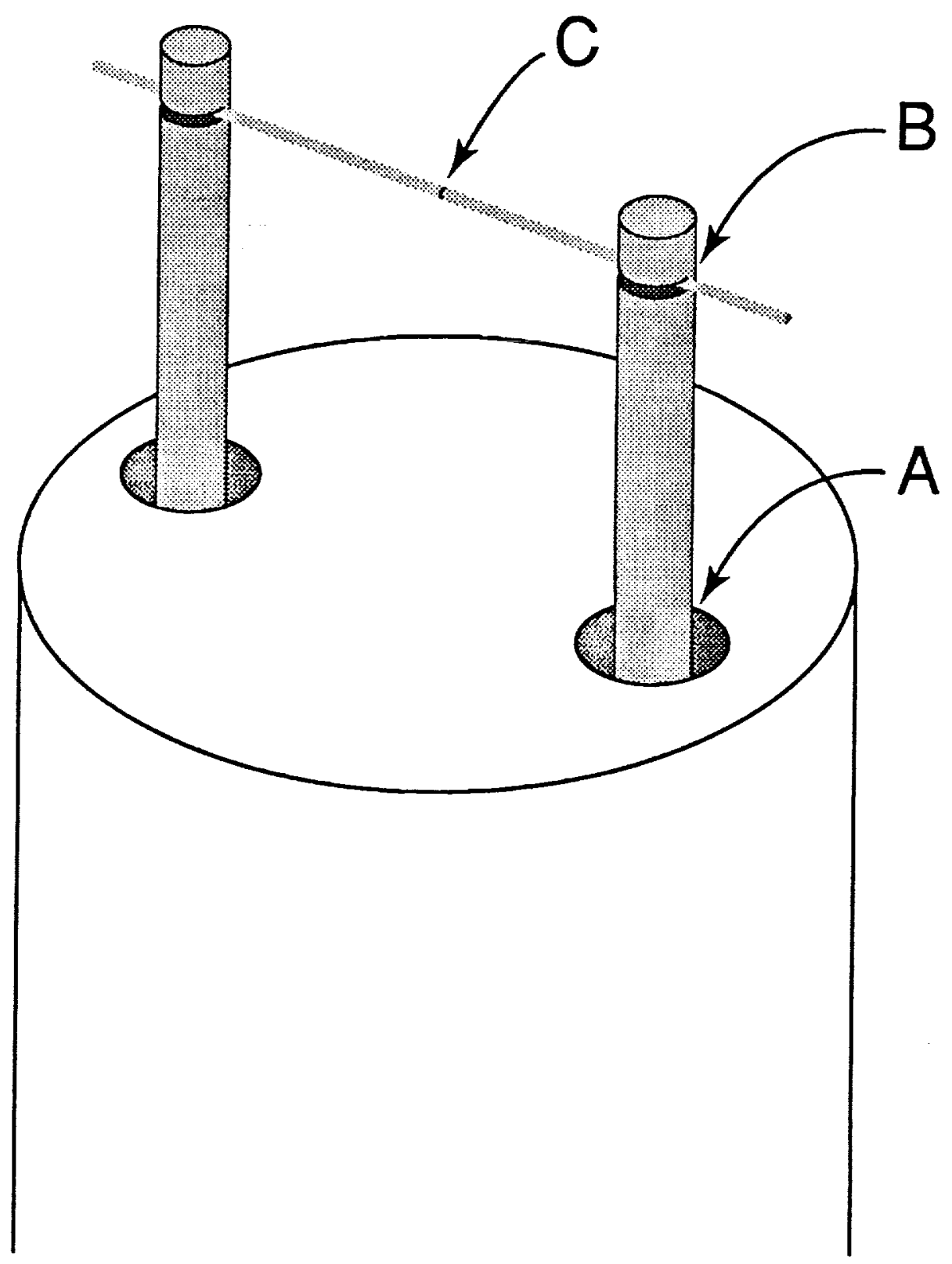

16. Schematic of test thermocouple 
FORGET . ALL

REAL DIM[ 1024 ] ARRAY TIME

COMPLEX DIM[ 1024 ] ARRAY TRANS

REAL DIM[ 6 ] ARRAY ANS

REAL DIM[ 1024 ] ARRAY ZMAGO

REAL DIM[ 1024 ] ARRAY ZARGO

INTEGER SCALAR NUM

REAL SCALAR MEN

REAL SCALAR CD

REAL DIM[ 1024 ] ARRAY FREQS

REAL DIM[ 1024 ] ARRAY SIGNAL

INTEGER dim[1024, 2 ] array DATA.BUFFER

1. $\mathrm{CD}:=$

.0000 DATA.BUFFER :=

LOAD . OVERLAY ACQUIS. SOV

.00001 .0000 A/D.TEMPLATE DEMO.TEMPLATE \DEFINE AN A/D TEMPLATE

DATA. BUFFER TEMPLATE. BUFFER

CYCLIC

2048.0000 TEMPLATE. REPEAT

CD CONVERSION.DELAY

DEMO. TEMPLATE A/D. INIT

$A / D$. IN $>$ ARRAY

LOAD. OVERLAY WAVEOPS. SOV

1024 REAL RAMP $1-2 *$ CD * 1000 / TIME := \SET TIME AXIS

DATA.BUFFER XSECT[ 1 ] 2048 -

\ CHANNEL 1 ON STACK (ANEMOMETER)

SIGNAL := SIGNAL

$-2048 / 10 *-55.274 * 353 .+$

SIGNAL := SIGNAL

0.5 SET. CUTOFF. FREQ

\ DECLARE ARRAY AS A TEMPLATE BUFFER

\ SET TEMPLATE BUFFER TO CYCLIC MODE

( SET REPETITIONS FOR I/O INSTRUCTION

( CONVERSION RATE (MSECS/SAMPLE)

( INITIALIZE CURRENT A/D TEMPLATE

$\backslash \mathrm{A} / \mathrm{D}$ INPUT TO TEMPLATES BUFFER

SMOOTH SIGNAL := SIGNAL

MEAN MEN := SIGNAL

MEN - SIGNAL := \ CENTER ON ORIGIN

TIME SUB[ $1,500,1]$ SIGNAL SUB[ 1,500,1]

XY.AUTO.PLOT'SIGNAL $\backslash$ PLOT CHANNEL 1

FFT

TRANS := TRANS ZMAG ZMAGO :=

\ TAKE FFT

5 SET.\#.POINTS

1 SET. \#.OPTIMA

ZMAGO SUB[ $1,120,1$ ] LOCAL.MAXIMA \ FIND INDEX AND MAX OF MAGNITUDE

SWAP NUM $:=$ NUM

$1-\mathrm{CD} / 1024$. $1000 * \mathrm{PI} *$

\INDEX OF MAX MAGNITUDE

FREQUENCY AT MAX MAGNITUDE

ANS $[1]:=$ ANS $[1]$ DROP ANS $[2]:=$

TRANS [ NUM ] DEG ZARG ANS [3 $]:=$ \ARGUMENT AT MAX MAGNITUDE

DATA.BUFFER XSECT[ 2 ] $2048-$

SIGNAL := SIGNAL

$124 *$ SIGNAL := SIGNAL

0.5 SET. CUTOFF. FREQ

SMOOTH SIGNAL := SIGNAL

MEAN MEN := SIGNAL
C CHANNEL 2 ON STACK (THERMOCOUPLE)

\ CALIBRATE (DEGREES F)

( SMOOTH DATA (CYCLES/POINT)

ASYST Version 3.00

Page 1 TEMP3.FOR 03/13/91 11:42:33.77 


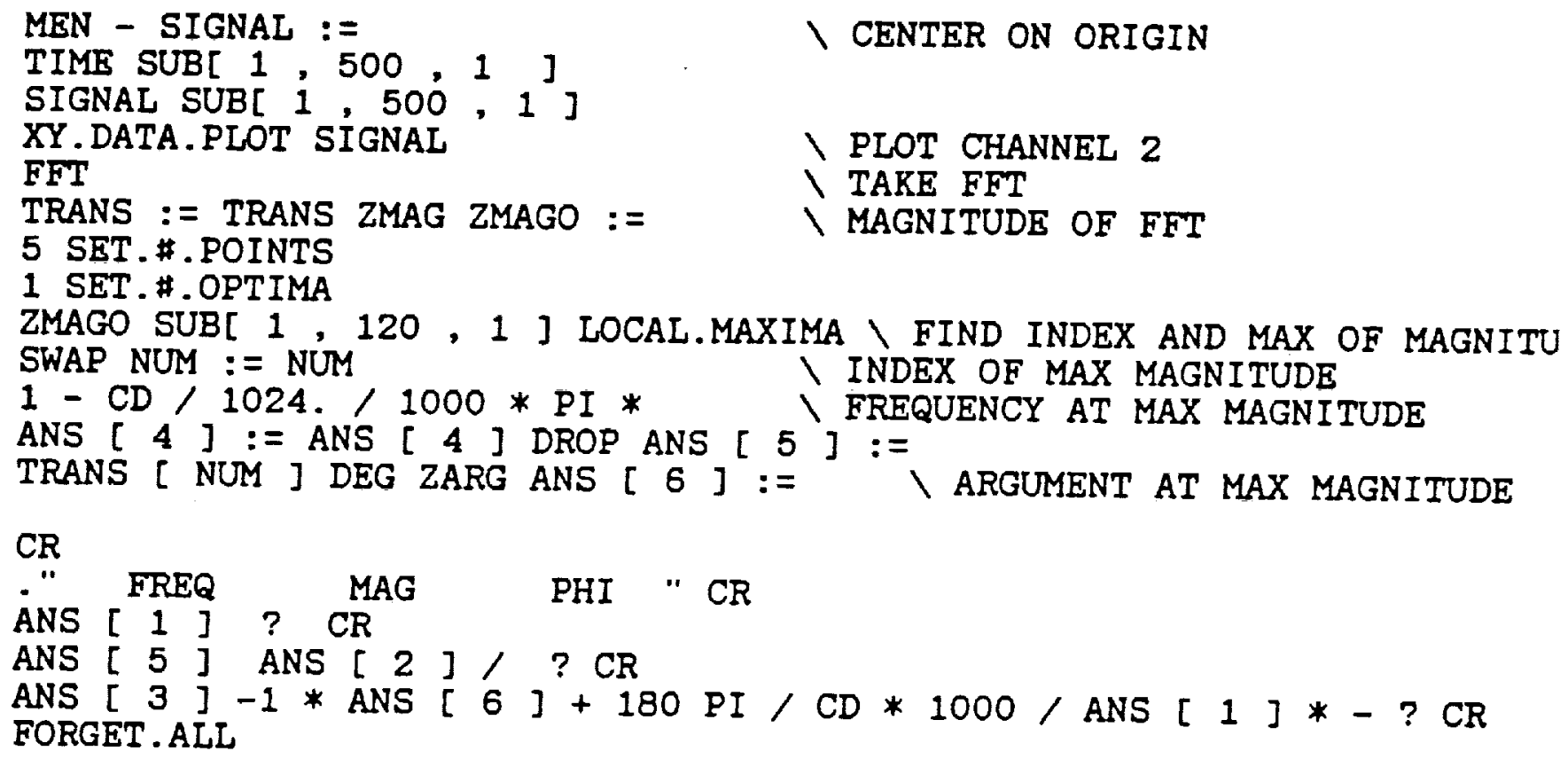

ASYST Version 3.00

Page 2 TEMP3.FOR 03/13/91 11:42:34.65

\section{ASYST software}




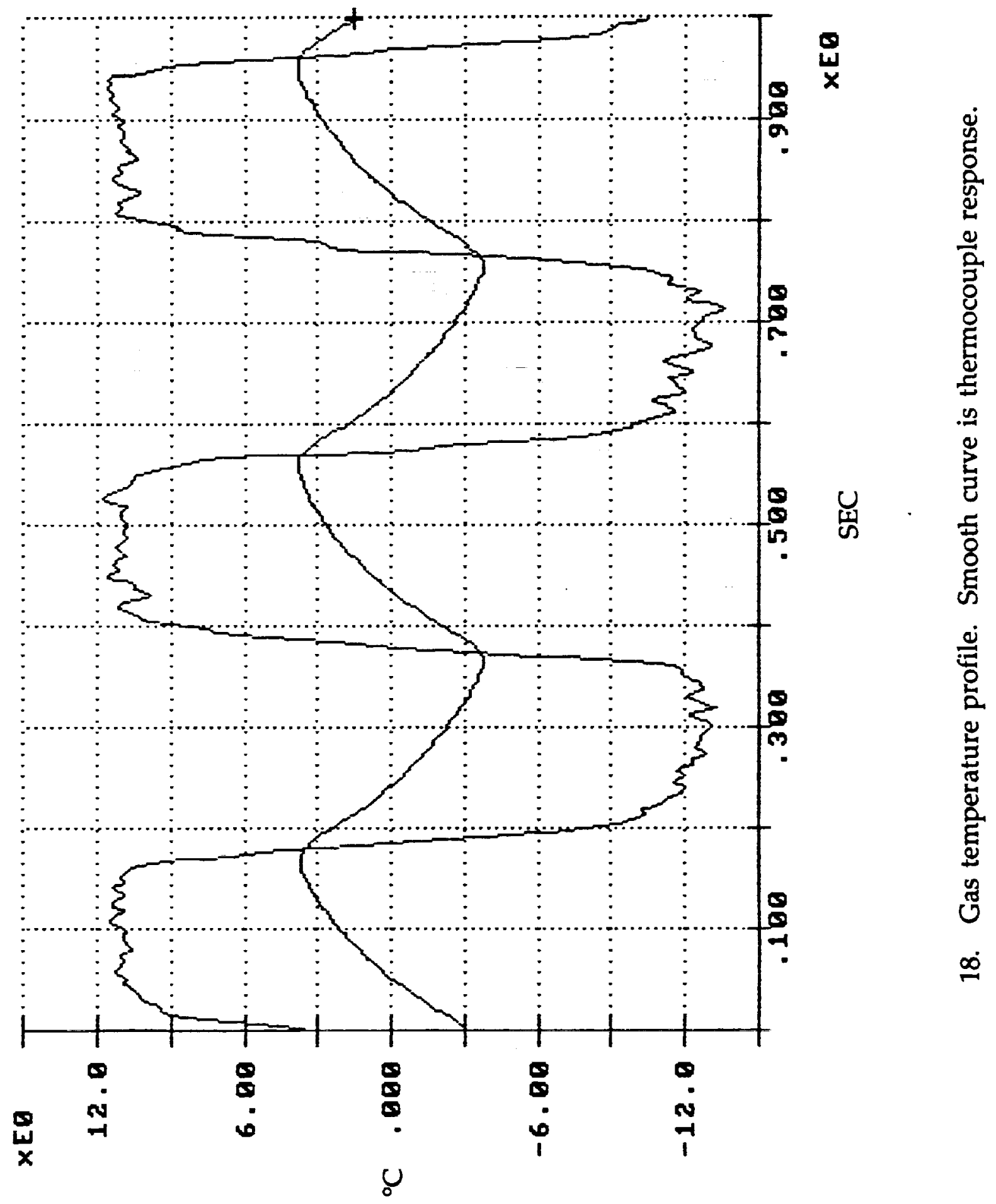




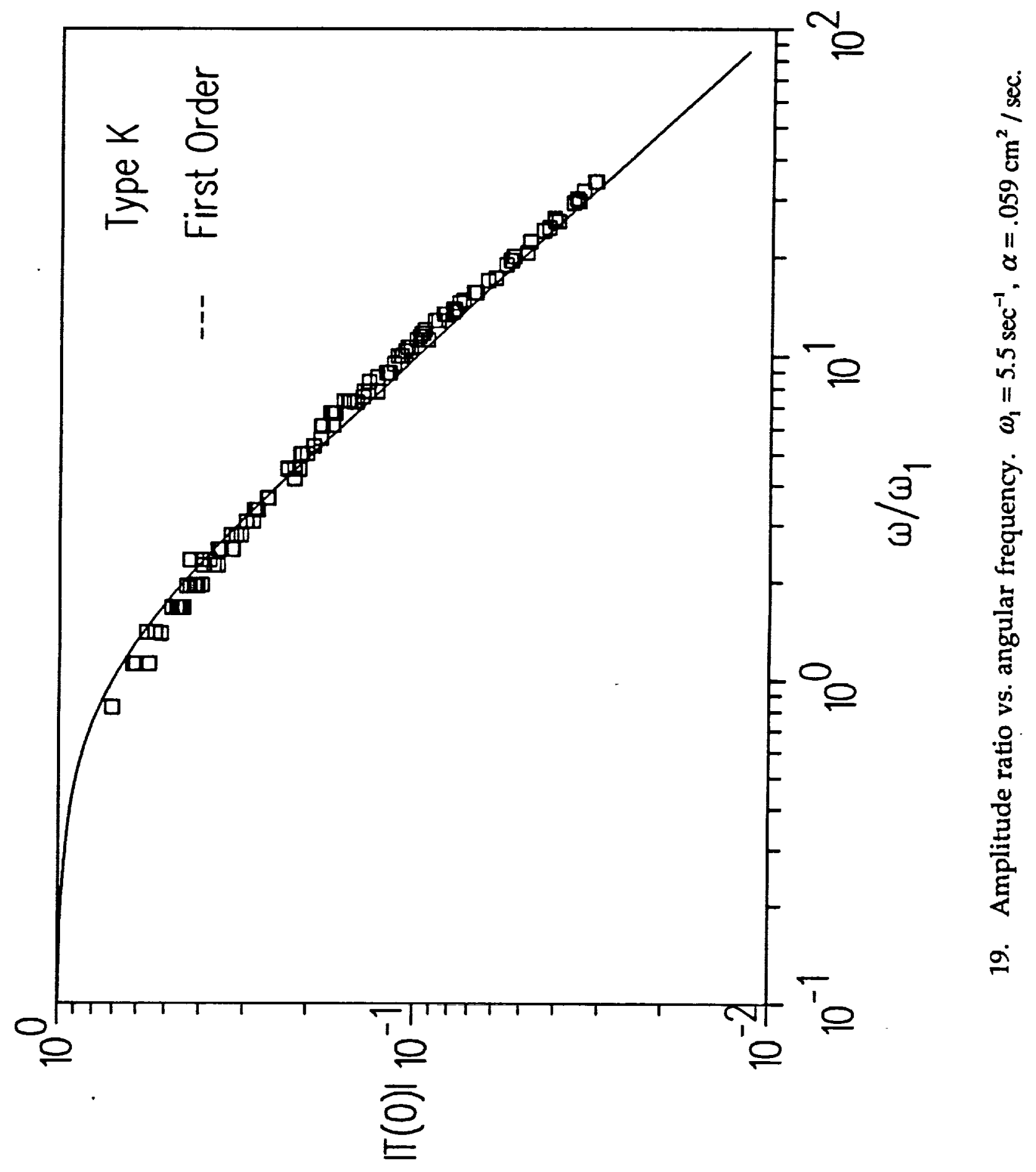




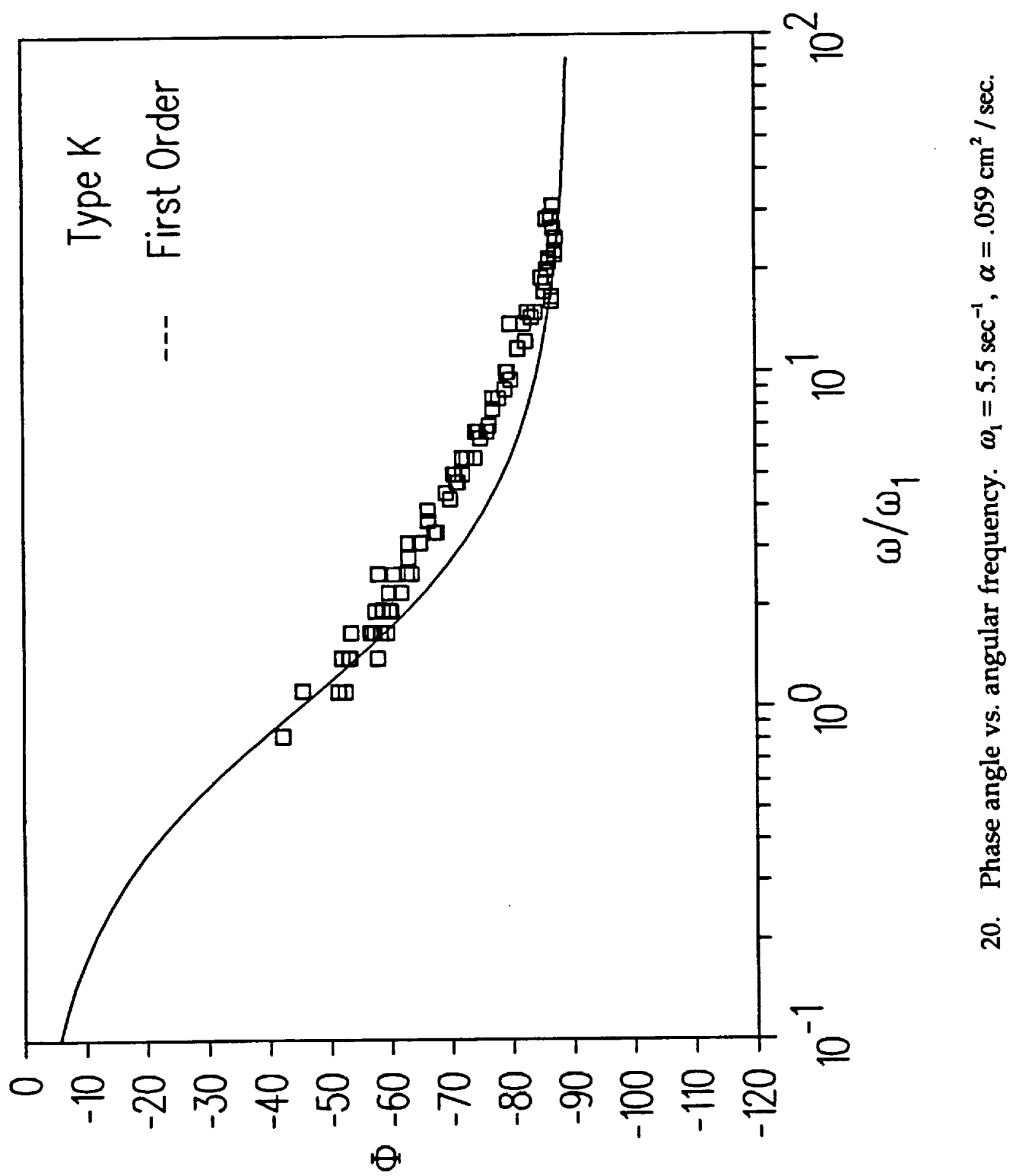




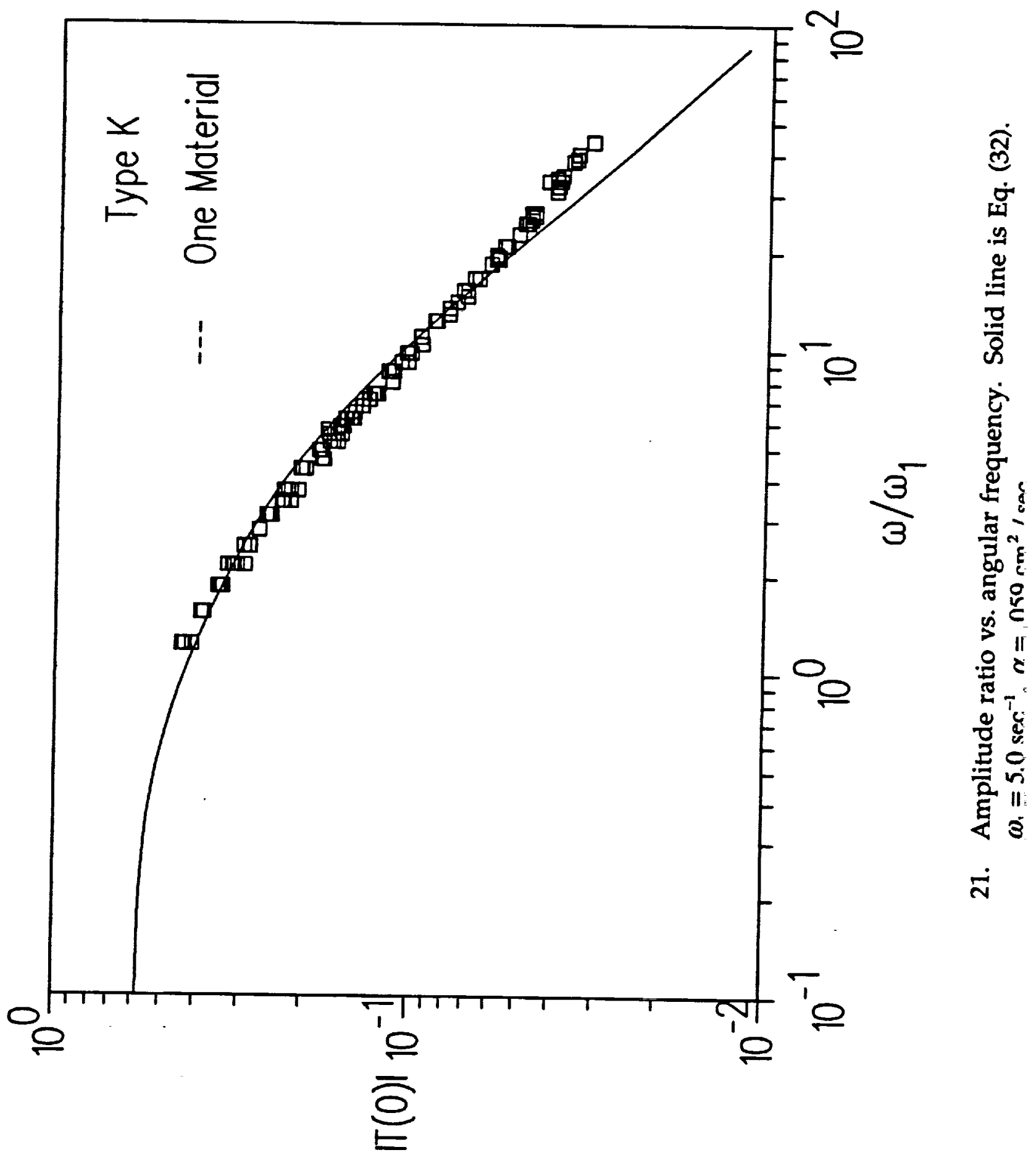

54 


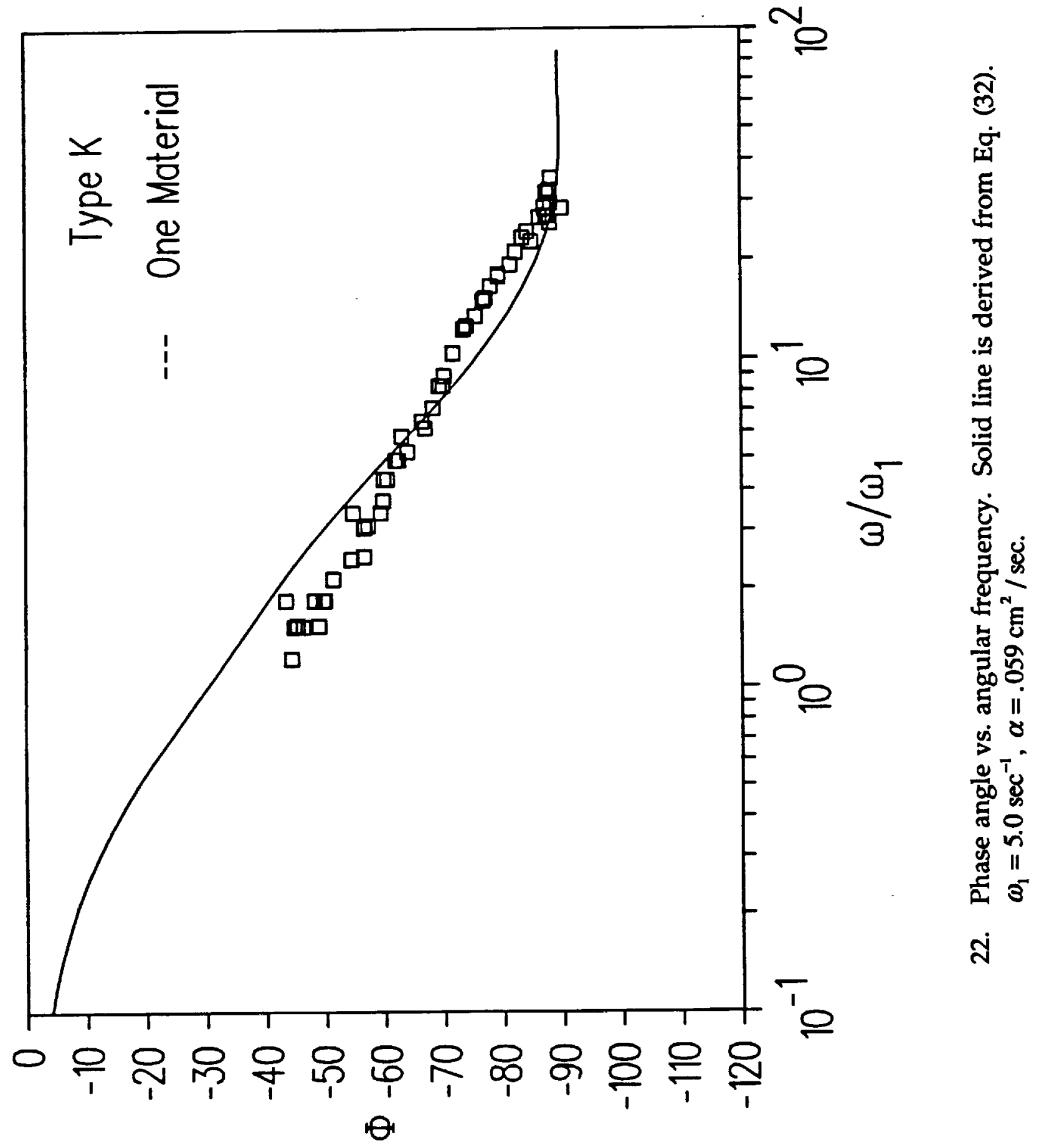


